\title{
Loss aversion with multiple investment goals
}

\author{
Enrico G. De Giorgi
}

Received: 11 July 2011 / Accepted: 10 November 2011 / Published online: 18 December 2011

C Springer-Verlag 2011

\begin{abstract}
This paper presents a time-continuous portfolio selection model with loss averse investors, who possess multiple investment goals at different time horizons. The model assumes partial narrow framing. Investors follow a two-step approach. First, they optimally allocate wealth among investment goals. Second, they determine an optimal investment strategy for each investment goal separately. We show that when loss aversion is according to the experimental findings, investors mainly invest their wealth to reach long-term goals and adopt investment strategies with high leverage to reach short-term goals. The overall strategy also display high leverage. The same patterns is observed when loss aversion is extreme and goals are very ambitious. By contrast, when loss aversion is extreme but goals are not too ambitious, investors mainly invest to reach short-term goals and adopt safe investment strategies for this purpose.
\end{abstract}

Keywords Loss aversion · Risk seeking - Mental accounting $\cdot$ Narrow framing · Portfolio selection

JEL Classification D10 - G11

\section{Introduction}

Recent studies have shown that the investment strategies of loss averse investors are very sensitive to the choice of the investment horizon. Benartzi and Thaler [5] show that loss averse investors with an investment horizon of less than one year prefer bonds to stocks, while the opposite holds for investment horizons larger than one year. Berkelaar et al. [6] derive the optimal strategy of loss averse investors and show that the proportion invested in stocks strictly increases as function of the investment horizon. Finally, Dierkes et al. [16] show that the attractiveness of investment strategies such as protective put or constant proportion

E. G. De Giorgi (凶)

Department of Economics, University of St. Gallen, Bodanstrasse 6, 9000 St. Gallen, Switzerland e-mail: enrico.degiorgi@unisg.ch 
portfolio insurance varies considerably with the investment horizon when investors possess preferences according to cumulative prospect theory (CPT) of Tversky and Kahneman [29].

These studies assume that investors possess a fixed reference point which corresponds to either a zero percent return level or the risk-free return. Moreover, investment strategies are evaluated according to gains and losses at the investment horizon. These assumptions exclude the case of investors who evaluate investment strategies according to different investment goals at possibly different time horizons, a typical case in goal-based approaches for asset allocation, which are quite popular in the wealth management industry [8-11,23,30].

This paper relaxes the usual assumptions on the specification of the reference point and the investment horizon and allows for the case where investors possess different investment goals (characterized by pre-defined target payoffs) at possibly different time horizons, which are then used as reference points. In our model investors are loss averse and possess an S-shaped value function on gains and losses, as in CPT. ${ }^{1}$

Investment strategies are evaluated according to gains and losses with respect to all goals. Specifically, investors are assumed to follow a two-stage approach. First, they optimally allocate wealth among their investment goals. Second, they derive the optimal investment strategy for each investment goal separately from their other goals, that is, investors narrowly frame their investment goals. Note, however, that narrow framing only occurs in the second stage of the investment process, while in the first stage investors consider all goals simultaneously. Therefore, our model only assumes "partial" narrow framing.

We show that loss averse investors with medium to high loss aversion mainly invest to reach long-term goals. Additionally, they adopt aggressive investment strategies with high leverage in order to reach short-term goals. The same follows when loss aversion is extreme and goals are too ambitious (that is, the initial wealth is too low relative to the discounted value of target payoffs). This result is due to the risk seeking behavior on losses. Risk seeking behavior on losses has first been reported by Kahneman and Tversky [20]. Also active stock traders seem to play negative-sum games, as reported by Statman [28]. Shefrin [26] argues that risk seeking behavior on losses coupled with ambitious investment goals offers an explanation for the observed high leverage ratios which contributed to the global financial crises erupted in 2008.

By contrast, when loss aversion is extreme and goals are not too ambitious (that is, the initial wealth is not too low relative to the discounted value of target payoffs), then a high proportion of the initial wealth is invested to reach short-term goals. Consequently, the probability to reach those goals is higher relative to medium- and long-term goals, that is, investors display a short-term perspective. Additionally, in this case, investors adopt safe investment strategies to reach short-term goals.

The concept of extreme loss aversion merits a brief discussion. Extreme loss aversion refers to investors, who reject any gain amount in exchange of an additional unit of loss (in a experimental setting, it is usually observed that people tend to accept an additional unit of loss in exchange of two-three additional units of gains). We use extreme loss aversion to describe investors, who are mainly concerned about reaching their investment goal. Suppose for example that an investor plan to buy the dream house worth one million dollars (target payoff) in five years. We expect such an investor to be very averse to scenarios where her goal is missed and to strongly prefer an investment that guarantees the target payoff almost surely. This behavior is consistent with extreme loss aversion, but not with moderate or even high loss aversion. Payne [24] and Diecidue and van de Ven [15] conjecture that the focus on the

1 Differently from CPT, our model does not include probability weighting. A formal analysis of the optimal portfolio allocation of investors with CPT preferences can be found in Jin and Zhou [19], Bernard and Ghossoub [7] and He and Zhou [17]. 
probability to reach a target payoff is stronger when the decision problem is more complex, as it is the case with many financial decisions. Diecidue and van de Ven [15] propose a model of preferences where the value function has a jump at the aspiration level, which implies extreme loss aversion. We argue that when investors' main objective is to meet predefined investment goals (for example, given target payoffs or benchmarks), extreme loss aversion might arise. Indeed, in this case, it is plausible to assume that ex ante the investor is mainly concerned about missing the target payoff, thus failing her goal. Obviously, ex post a surplus above the target payoff is positively valued. However, if the investor would require a surplus ex ante, then she would adapt her target payoff in the first place, that is, aim to a more ambitious investment goal.

The model presented in this paper is related to the behavioral portfolio theory (BPT) of Shefrin and Statman [27]. However, beside the fact that BPT is build on the SP/A theory of Lopes [22], our model differs from BPT in at least two directions. ${ }^{2}$ First, our model is a continuous-time model, while BPT is a static model. This allows us to consider investment goals at different horizons. Second, in this paper how investors' wealth is allocated among investment goals is determined endogenously, while in BPT this is exogenously given.

Mental accounting in a mean-variance framework has been studied by Das et al. [12]. Their model is static and how wealth is allocated among investment goals is exogenously given. While Das et al. [12] assume mean-variance preferences, they derive investors' aversion to volatility from the probability to reach a target payoff, thus in their model it is assumed that investors focus on the probability of success. The main result in Das et al. [12] is that in their setting, when short-sale is allowed, portfolios that follows from mean-variance analysis with mental accounting belong to the mean-variance efficient frontier, that is, mental accounting does not introduce inefficiency. We show that a similar result holds in our model when loss aversion is extreme.

As we discussed above, this paper also relates to Benartzi and Thaler [5], Berkelaar et al. [6] and Dierkes et al. [16]. Differently from these papers, here we do not address the question about how the investment strategies of loss averse investors change with the time horizon, but we ask the question about how loss averse investors with several investments goals (at different horizons) allocate their wealth among them. However, the theoretical setting used to address this question is similar to Berkelaar et al. [6].

Finally, our paper relates to Barberis and Huang [1] , Barberis et al. [4], Barberis and Huang [2,3], and De Giorgi and Legg [13], who present consumption-portfolio models with CPT preferences and narrow framing. In these papers it is assumed that at each point in time investors narrowly frame single stocks, that is, investor's utility not only depend on the overall wealth's distribution, but also on the payoff distribution of single stocks. Therefore, in these papers narrow framing refers to single assets or a subclass of assets. By contrast, in our model narrow framing refers to investment goals, which are pursued by all available assets, and our objective is to compare payoff distributions at different horizons. We refer to Hens and Bachmann [18, Sect. 8.6], who distinguish between mental accounting and goal-based approach, the former being related to the case where investors narrowly frame single assets or a of subclass of assets, while the latter occurs when investors narrowly frame specific investment goals, as in this paper.

The remainder of the paper is structured as follows. Section 2 introduces the model and presents the main theoretical results. Section 3 provides numerical examples to illustrate our results. Section 4 concludes. All proofs are given in the Appendix.

${ }^{2}$ As discussed above, our model includes two main features of CPT, that is, loss aversion and an S-shaped value function. By contrast, SP/A theory assumes a concave value function. For a rigorous comparison of CPT and SP/A theory, see [25]. 


\section{The investment model}

\subsection{The economy}

We assume a standard, continuous-time financial market as described in Karatzas and Shreve [21]. There are $K+1$ assets with price $S_{k}(t)$ at time $t$ for $k=0, \ldots, K$. The zero-th asset is a risk-free asset $S_{0}(t)$ :

$$
d S_{0}(t)=r(t) S_{0}(t) d t
$$

where $r(\cdot)$ is the interest rate process. The remaining assets are risky and follow an Ito process

$$
d S_{k}(t)=\mu_{k}(t) S_{k}(t) d t+S_{k}(t) \sum_{l=1}^{K} \sigma_{k l}(t) d B_{l}(t), \quad k=1, \ldots, K
$$

with drift rates $\mu_{k}(\cdot)$ and volatility components $\sigma_{k l}(\cdot)$. The processes $r(\cdot), \mu(\cdot)=$ $\left(\mu_{1}, \ldots, \mu_{K}\right)$ and $\sigma(\cdot)=\left(\sigma_{k l}\right)_{k, l=1, \ldots, K}$ are progressively measurable with respect to the filtration generated by the $K$-dimensional Browniam motion $B=\left(B_{1}, \ldots, B_{K}\right)^{\prime}$ and satisfy the usual regularity conditions [21, Definition 1.3].

We assume a complete market. This is the case when the volatility matrix $\sigma(t)$ has full rank for all $t$ and there exists a $K$-dimensional progressively measurable process $\kappa$ such that $\sigma(t) \kappa(t)=\mu(t)-\mathbf{1} r(t)$ almost surely for all $t$, where $\mathbf{1}$ is a $K$-dimensional vector of 1 . Under market completeness, there exists a unique pricing kernel $\xi$ :

$$
\xi(t)=\exp \left(-\int_{0}^{t}\left(r(s)+\frac{1}{2}\|\kappa(s)\|^{2}\right) d s-\int_{0}^{t} \kappa(s)^{\prime} d B(s)\right) .
$$

$\kappa$ is called the market price of risk process. The pricing kernel satisfies the following dynamics:

$$
\frac{d \xi_{t}}{\xi_{t}}=-r(t) d t-\kappa(t)^{\prime} d B(t), \quad \xi(0)=\xi_{0}=1
$$

\subsection{Investor's preferences}

The investor possesses $J$ different investment goals, which can be characterized by target payoffs $\bar{W}_{j}$ the investor wants to obtain at time $T_{j}$, for $j=1, \ldots, J .^{3}$ At time $t$ she allocates a fraction $w_{j}(t)$ of her wealth $W(t)$ to goal $j$ and chooses goal-specific portfolios $\lambda_{j}(t)=\left(\lambda_{j, 1}(t), \ldots, \lambda_{j, K}(t)\right)^{\prime}$, where $\lambda_{j, k}(t)$ is the fraction of wealth $W_{j}(t)=w_{j}(t) W(t)$ allocated to the asset $k$ at time $t$. We put $W_{0}=W(0)$ and $w_{j, 0}=w_{j}(0)$. The wealth dynamics for goal $j$ is given as follows:

$$
\begin{gathered}
d W_{j}(t)=r(t) W_{j}(t) d t+(\mu(t)-\mathbf{1} r(t))^{\prime} \lambda_{j}(t) W_{j}(t) d t+\sigma(t)^{\prime} \lambda_{j}(t) W_{j}(t) d B(t), \\
W_{j}(0)=w_{j, 0} W_{0} .
\end{gathered}
$$

3 Target payoffs are assumed to be exogenously given and fixed. However, we could easily extends our model to allow target payoffs to be endogenous and stochastic. For example, if the target wealth for goal $j$ is the value at time $T_{j}$ of a benchmark portfolio $\lambda_{b}(t)$, then we simply take the difference $W_{j}(t)-W_{b}(t)$ where $W_{b}(t)$ is the wealth level at time $t$ obtained when the initial wealth $W_{j}(0)$ is invested in $\lambda_{b}(t)$. Since $W_{j}(t)-W_{b}(t)$ also follows an Ito process, we can apply the same technology to extend our model to the case where target payoffs are endogenous and stochastic; see [19]. 
Therefore, the terminal wealth available for gaol $j$ at the horizon $T_{j}$ is stochastic and given by $W_{j}\left(T_{j}\right)$. The investor derives utility from each goal separately at the corresponding horizons $T_{j}$. This implies that the covariances between goal-specific portfolios are ignored and wealths $W_{j}\left(T_{j}\right)$ are fully consumed at time $T_{j}$, even if the investor is not able to reach her goal $\left(W_{j}\left(T_{j}\right)<\bar{W}_{j}\right)$ or there exists a surplus $\left(W_{j}\left(T_{j}\right)>\bar{W}_{j}\right)$. In our model we will see that in case $W_{j}\left(T_{j}\right)<\bar{W}_{j}$, then $W_{j}\left(T_{j}\right)=0$, that is, there is nothing to consume relative to goal $j$ when goal $j$ is not reached.

The choice function for goal $j$ is equal

$$
V_{j}\left(W ; \bar{W}_{j}\right)=\int_{-\infty}^{\infty} v_{j}\left(x-\bar{W}_{j}\right) d\left(F_{W}(x)\right)
$$

where $v_{j}(x)$ is the piecewise-power value function

$$
v_{j}(x)= \begin{cases}\beta_{j}^{+} x^{\alpha_{j}}, & x>0 \\ -\beta_{j}^{-}(-x)^{\alpha_{j}}, & x \leq 0\end{cases}
$$

with $\beta_{j}^{-} \geq \beta_{j}^{+} \geq 0$ and $\alpha_{j} \in(0,1) . F_{W}$ denotes the cumulative distribution function of the random payoff $W$.

The value function $v_{j}$ is S-shaped as in CPT. The two parameters $\beta_{j}^{+}$and $\beta_{j}^{-}$characterize loss aversion, which is measured by the parameter $\beta_{j}=\beta_{j}^{-} / \beta_{j}^{+}$, which we call the degree of loss aversion (see also [6]). We say that an investor is extremely loss averse for investment goal $j$ when $\beta_{j} \rightarrow \infty$, that is, $\beta_{j}^{+} \rightarrow 0$ and $\beta_{j}^{-}>0$. Extreme loss aversion occurs when the investor does not obtain any positive value for being above the target wealth of gaol $j$, but only a negative value for being below it, that is, she is only concerned about reaching the investment goal, as discussed in the Introduction.

\subsection{Investor's decision problem}

Given her preferences as described in the previous section, the investor determines at each time $t$ how to optimally split wealth among the different investment goals and, additionally, how to optimally invest the wealth amounts allocated to the different investment goals. She solves the following decision problem:

$$
\begin{aligned}
& \max _{\lambda_{1}(t), \ldots, \lambda_{J}(t)} \sum_{j=1}^{J} \delta^{-T_{j}} V_{j}\left(W_{j}\left(T_{j}\right) ; \bar{W}_{j}\right) \\
& \text { such that for } j=1, \ldots, J \\
& d W_{j}(t)=r(t) W_{j}(t) d t+(\mu(t)-\mathbf{1} r(t))^{\prime} \lambda_{j}(t) W_{j}(t) d t+\sigma(t)^{\prime} \lambda_{j}(t) W_{j}(t) d B(t)(6) \\
& W_{j}(t) \geq 0, t \in\left[0, T_{j}\right], \\
& \sum_{j=1}^{J} W_{j}(0)=W_{0} .
\end{aligned}
$$

The constant $\delta \geq 1$ is the discount factor and characterizes the investor's time preferences, that is, her preference for immediate consumption relative to consumption far in the future.

We apply martingale methods and rewrite the dynamic decision Problem (6) as a static one: 


$$
\begin{aligned}
& \max _{\substack{W_{1}\left(T_{1}\right), \ldots, W_{J}\left(T_{J}\right) \\
w_{1,0}, \ldots, w_{J, 0}}} \sum_{j=1}^{J} \delta^{-T_{j}} \mathbb{E}\left[v_{j}\left(W_{j}\left(T_{j}\right)-\bar{W}_{j}\right)\right] \\
& \text { such that } \\
& \mathbb{E}\left[\xi\left(T_{j}\right) W_{j}\left(T_{j}\right)\right] \leq \xi_{0} w_{j, 0} W_{0}, \\
& W_{j}\left(T_{j}\right) \geq 0, w_{j, 0} \geq 0, \quad j=1, \ldots, J, \\
& \sum_{j=1}^{J} w_{j, 0} \leq 1 .
\end{aligned}
$$

The vector $w_{0}=\left(w_{1,0}, \ldots, w_{J, 0}\right)^{\prime}$ corresponds to the wealth's shares at time $t=0$. At time $t=0$ the investor decides how to split wealth among the $J$ investment goals. Consequently, she allocates $W_{j}(0)=w_{j, 0} W_{0}$ to goal $j$, which corresponds to the budget constraint for this goal. Initial wealth shares and the corresponding goal-specific terminal wealths determine the investor's global value she obtains from the $J$ different investment goals.

Since the objective function is additively separable among the various investment goals, we can solve Problem (7) in two stages (see [14]). First, for a given vector of initial wealth shares $w_{0}=\left(w_{1,0}, \ldots, w_{J, 0}\right)^{\prime}$, we solve for each investment goal $j$ the following goal-specific problem:

$$
\begin{aligned}
& \max _{W_{j}\left(T_{j}\right)} \mathbb{E}\left[v_{j}\left(W_{j}\left(T_{j}\right)-\bar{W}_{j}\right)\right] \\
& \text { such that } \\
& \mathbb{E}\left[\xi\left(T_{j}\right) W_{j}\left(T_{j}\right)\right] \leq \xi_{0} w_{j, 0} W_{0}, \\
& W_{j}\left(T_{j}\right) \geq 0 .
\end{aligned}
$$

The optimal terminal wealths obviously depend on $w_{0}$ since wealth shares at time 0 define the budget constraints for all investment goals. Second, given optimal terminal wealths $W_{j}^{\star}\left(T_{j}\right)$ for all goals as function of $w_{0}$, we find the optimal vector of shares $w_{0}^{\star}$ that maximizes the investor's value function:

$$
\begin{aligned}
& \max _{w_{1,0}, \ldots, w_{J, 0}} \sum_{j=1}^{J} \delta^{-T_{j}} \mathbb{E}\left[v_{j}\left(W_{j}^{\star}\left(T_{j}\right)-\bar{W}_{j}\right)\right] \\
& \text { such that } \\
& w_{j, 0} \geq 0, j=1, \ldots, J \\
& \sum_{j=0}^{J} w_{j, 0} \leq 1 .
\end{aligned}
$$

We point out that in our model, goals' specific portfolios are somehow related, even if the portfolio decision problem for each goal does not directly account for the investment strategies of the other goals. This can be seen as follows: if one goal is very ambitious or very important so that it is optimal for the investor to put a high proportion of her wealth on it, then less wealth will be available for other goals and the investors might use aggressive investment strategies (given her risk seeking behavior on losses) for them. Therefore, the investor might finally end up with aggressive investment strategies for almost all goals, while she safely invest to reach few important goals.

The following Proposition, which essentially corresponds to Proposition 1 in Berkelaar et al. [6], gives the solution to Problem (8): 
Proposition 2.1 Let $w_{0}=\left(w_{1,0}, \ldots, w_{J, 0}\right)^{\prime}$ be a vector of initial wealth shares. Then for $j=1, \ldots, J$ the optimal terminal wealth for investment goal $j$ is given by:

$$
W_{j}^{\star}\left(T_{j}\right)=\left\{\begin{array}{cc}
\bar{W}_{j}+\left(\frac{y_{j} \xi\left(T_{j}\right)}{\beta_{j}^{+} \alpha}\right)^{1 /\left(\alpha_{j}-1\right)} & \text { if } \xi\left(T_{j}\right)<\xi_{j}^{\star}\left(y_{j}\right), \\
0, & \text { if } \xi\left(T_{j}\right) \geq \xi_{j}^{\star}\left(y_{j}\right),
\end{array}\right.
$$

where $\xi_{j}^{\star}\left(y_{j}\right)$ solves $g_{j}\left(\xi_{j}^{\star}\left(y_{j}\right), y_{j}\right)=0$ and $y_{j} \geq 0$ satisfies $\mathbb{E}\left[\xi\left(T_{j}\right) W_{j}^{\star}\left(T_{j}\right)\right]=$ $\xi_{0} w_{j, 0} W_{0}$. The function $g_{j}$ is defined as follows:

$$
g_{j}(x, y)=\frac{1-\alpha_{j}}{\alpha_{j}}\left(\frac{1}{y x}\right)^{\alpha_{j} /\left(1-\alpha_{j}\right)}\left(\beta_{j}^{+} \alpha_{j}\right)^{1 /\left(1-\alpha_{j}\right)}-\bar{W}_{j} y x+\beta_{j}^{-} \bar{W}_{j}^{\alpha_{j}}
$$

where $x, y>0$.

Optimal terminal wealths $W_{j}^{\star}\left(T_{j}\right)$ present the following characteristic. In good states of the world at time $T_{j}\left(\xi\left(T_{j}\right)<\xi_{j}^{\star}\left(y_{j}\right)\right)$ the investor is able to reach her investment goal $\bar{W}_{j}$. In this case there is a strictly positive surplus $\left(\beta_{j}^{+} \alpha_{j} /\left(y_{j} \xi_{j}\left(T_{j}\right)\right)\right)^{1 /\left(1-\alpha_{j}\right)}$ which increases as $\xi_{j}\left(T_{j}\right)$ becomes smaller. By contrast, in bad states of the world at time $T_{j}\left(\xi\left(T_{j}\right) \leq \xi_{j}^{\star}\left(y_{j}\right)\right)$ the investor fails to reach her goal and her terminal wealth for this goal is zero. The probability of beating the investment goal corresponds to the probability that $\xi\left(T_{j}\right) \leq \xi^{\star}\left(y_{j}\right)$. We will provide an explicit expression for this probability under further assumptions on the dynamics of the pricing kernel $\xi_{t}$.

In order to derive optimal initial wealth shares $w_{0}^{\star}$, we need to understand how $W_{j}^{\star}\left(T_{j}\right)$ depends on $w_{0}$. For sake of simplicity, we drop the index $j$ in our discussion below, since the results apply to all investment goals. When it is not confusing we denote by $w_{0}$ the wealth share allocated to one specific investment goal. The following Lemma provides an explicit characterization of $\xi^{\star}(y)$.

Lemma 2.1 Let $x, y>0$ and $g: \mathbb{R}_{+}^{2} \rightarrow \mathbb{R}$ be defined as in Eq. 11. Then for $y>$ $0, g(x, y)=0$ possesses a unique solution $\xi^{\star}(y)=a / y$ where $a>0$ solves:

$$
\frac{1-\alpha}{\alpha} a^{-\alpha /(1-\alpha)}\left(\beta^{+} \alpha\right)^{1 /(1-\alpha)}-a \bar{W}+\beta^{-} \bar{W}^{\alpha}=0 .
$$

We now impose additional conditions on the dynamics of the pricing kernel $\xi_{t}$. We assume that the interest rate process $r$, the drift process $\mu$ and the volatility matrix $\sigma$ are constant. ${ }^{4}$

Let $m=-\left(r+(1 / 2)\|\kappa\|^{2}\right)$ and $s^{2}=\|\kappa\|^{2}$. Then $\xi(T)$ is log-normally distributed with parameters $m_{T}=m T$ and $s_{T}=s \sqrt{T}$. Under these conditions, we can easily determine the probability of reaching an investment goal at the time horizon $T$, which corresponds to $\Phi\left(\left(\log \left(\xi^{\star}(y)-m_{T}\right) / s_{T}\right)\right)$. We also obtain an explicit characterization of the parameter $y$ which satisfies the budget constraint for $W^{\star}(T)$ in Prosition 2.1.

Lemma 2.2 Let $W^{\star}(T)$ be the optimal wealth from Proposition 2.1. Then $y>0$ solves

$$
\mathbb{E}\left[\xi(T) W^{\star}(T)\right]=\xi_{0} w_{0} W_{0}
$$

if and only if $y>0$ solves $h(y)=w_{0}$ where:

$$
h(y)=b \Phi\left(\frac{\log (a / y)-m_{T}-s_{T}^{2}}{s_{T}}\right)+c y^{\frac{1}{\alpha-1}} \Phi\left(\frac{\log (a / y)-m_{T}-\frac{\alpha}{\alpha-1} s_{T}^{2}}{s_{T}}\right) .
$$

4 This assumption is for simplicity of the exposition. Our analysis could be easily extended to the case where $r, \mu$ and $\sigma$ are time-varying and deterministic. 
The constant $a>0$ solves Eq. 12 from Lemma 2.1. Additionally, $b=\frac{\bar{W}}{\xi_{0} W_{0}} \exp (-r T)$ and $c=\frac{1}{\xi_{0} W_{0}}\left(\beta^{+} \alpha\right)^{\frac{1}{1-\alpha}} \exp \left(\frac{\alpha m_{T}}{\alpha-1}+\frac{1}{2} \frac{\alpha^{2} s_{T}^{2}}{(\alpha-1)^{2}}\right)$.

The function $h$ is strictly decreasing. Therefore $y$ decreases as the initial share allocated to one investment goal increases. Consequently, $\xi^{\star}$ becomes larger, that is, the probability of reaching the investment goal is higher as more wealth is allocated to that investment goal. Moreover, for $y$ and $\beta^{-}$fix, $h(y)$ strictly increases as $\beta^{+}$increases, that is, as loss aversion decreases. Consequently, $y$ decreases with loss aversion, that is, as loss aversion increases, the probability of reaching the investment goal increases, but the surplus becomes smaller. The probability of reaching the investment goal is therefore maximal when $\beta^{+}=0$, which implies extreme loss aversion.

When $h(y)=w_{0}$ possesses a solution, then it is unique since $h$ is strictly decreasing. For $\beta^{+}>0, h(y)=w_{0}$ possesses a solution for all $w_{0}$. However, for $\beta^{+}=0, h(y)=w_{0}$ cannot be solved for $w_{0}>b$ since $h(y) \in[0, b]$ for all $y \geq 0$. For the case $\beta^{+}=0$ we impose some further conditions on $b$ when we solve for the vector of wealth shares $\left(w_{1,0}, \ldots, w_{J, 0}\right)^{\prime}$. If $\beta^{+}=0$ and $w_{0} \leq b$, from Eq. 13 we obtain an explicit solution for $y$ as function of $w_{0}$ :

Corollary 2.1 Let $h$ be as given by Eq. 13. Let $\beta^{+}=0$ and $w_{0} \leq b$. Then $h(y)=w_{0}$ if and only if

$$
y=a \exp \left(-s_{T} \Phi^{-1}\left(w_{0} / b\right)-m_{T}-s_{T}^{2}\right),
$$

where $b=\frac{\bar{W}}{\xi_{0} W_{0}} \exp (-r T)$.

Note that for $\beta^{+}=0$, the optimal terminal wealth $W_{T}^{\star}$ does not depend on $\beta^{-}$. Indeed, $\beta^{-}$only enters into $\xi^{\star}(y)$ through the constant $a$. However, since $\xi^{\star}(y)=a / y$ we have $\xi^{\star}(y)=\exp \left(s_{T} \Phi^{-1}\left(w_{0} / b\right)+m_{T}+s_{T}^{2}\right)$, which is independent from $a$. More generally, the following result holds:

Corollary 2.2 The optimal terminal wealth $W^{\star}(T)$ depends on $\beta^{+}$and $\beta^{-}$only trough the ratio $\beta^{-} / \beta^{+}$, that is, the degree of loss aversion.

The following Lemma gives an explicit characterization of the optimal utility level $\mathbb{E}\left[W^{\star}(T)\right]$ as function of $y$ :

Lemma 2.3 We have $\mathbb{E}\left[W^{\star}(T)\right]=k(y)$ where

$$
k(y)=\bar{W} \Phi\left(\frac{\log (a / y)-m_{T}}{s_{T}}\right)+d y^{\frac{1}{\alpha-1}} \Phi\left(\frac{\log (a / y)-m_{T}+\frac{s_{T}^{2}}{1-\alpha}}{s_{T}}\right) .
$$

The constant $a>0$ solves Eq. 12 from Lemma 2.1 and $d=\left(\beta^{+} \alpha\right)^{\frac{1}{1-\alpha}} \exp \left(\frac{m_{T}}{\alpha-1}+\frac{1}{2} \frac{s_{T}^{2}}{(\alpha-1)^{2}}\right)$. The function $k$ is continuous, strictly decreasing and $\lim _{y \rightarrow \infty} k(y)=0$.

Note that

$$
k(y)=\xi_{0} W_{0} \exp (r T) h\left(y \exp \left(-s_{T}^{2}\right)\right),
$$

where $h$ is defined in Lemma 2.2. We now rewrite Problem (9) as follows. Optimal wealths $W_{1}\left(T_{1}\right), \ldots, W_{J}\left(T_{J}\right)$ are given by Proposition 2.1 where $y_{j}=h^{-1}\left(w_{j, 0}\right)$ for $j=1, \ldots, J$ and $w_{0}=\left(w_{1,0}, \ldots, w_{J, 0}\right)^{\prime}$ solves 


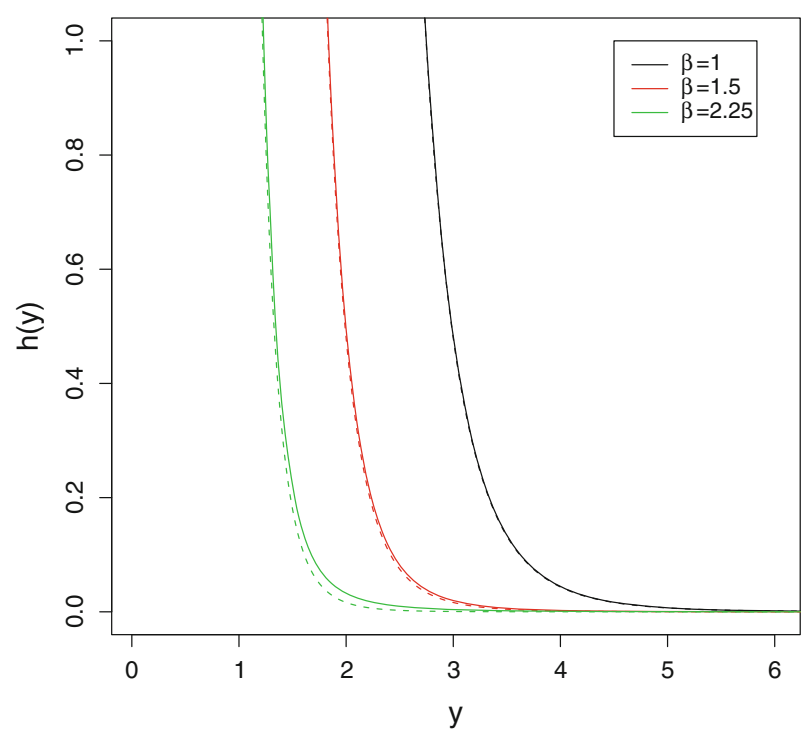

Fig. 1 Budget function $h$ from Lemma 2.2 (full line) and its approximation $c y^{\frac{1}{\alpha-1}}$ (dashed line) for $\beta^{+}=$ $1,1.5,2.25$ and $\beta^{-}=2.25$ (that is, the degree of loss aversion is $\beta=\beta^{-} / \beta^{+}=2.25,1.5,1$, respectively). We assumed $\alpha=0.88, T=10, r=0.03, \delta=\log (r)$ and $\kappa=0.2$

$$
\begin{aligned}
& \max _{w_{1,0}, \ldots, w_{J, 0}} \sum_{j=1}^{J} \delta^{-T_{j}} \exp \left(r T_{j}\right) h_{j}\left(h_{j}^{-1}\left(w_{j, 0}\right) \exp \left(-s_{T_{j}}^{2}\right)\right) \\
& \text { such that } \\
& w_{j, 0} \geq 0 \text { for } j=1, \ldots, J \\
& \sum_{j=1}^{J} w_{j, 0}=1
\end{aligned}
$$

In general, Problem (16) must be solved numerically, since no explicit expression for $h_{j}^{-1}$ is available. We present some numerical exercises in Sect. 3. Note, however, that for $y>0$ large enough and $y \approx 0$, we have

$$
h_{j}(y) \approx c_{j} y^{\frac{1}{\alpha_{j}-1}}
$$

where $c_{j}=\frac{1}{\xi_{0} W_{0}}\left(\beta_{j}^{+} \alpha_{j}\right)^{\frac{1}{1-\alpha_{j}}} \exp \left(\frac{\alpha_{j} m_{T_{j}}}{\alpha_{j}-1}+\frac{1}{2} \frac{\alpha_{j}^{2} s_{T_{j}}^{2}}{\left(\alpha_{j}-1\right)^{2}}\right)$. This approximation also holds for all $y>0$ when $\beta_{j}^{+}$is large enough (or, the degree of loss aversion $\beta_{j}=\beta_{j}^{-} / \beta_{j}^{-}$is small enough) and the discounted value of the target wealth $\bar{W}_{j} \exp \left(-r T_{j}\right)$ is not too high relative to the initial wealth; see Fig. 1. In this case, for the term in the objective function of Problem (16) corresponding to goal $j$ we obtain

$$
\begin{aligned}
& \delta^{-T_{j}} \exp \left(r T_{j}\right) h_{j}\left(h_{j}^{-1}\left(w_{j, 0}\right) \exp \left(-s_{T_{j}}^{2}\right)\right) \\
& \approx w_{j, 0} \delta^{T_{j}} \exp \left(r T_{j}\right) \exp \left(\frac{1}{1-\alpha_{j}} s_{T_{j}}^{2}\right) \\
& =w_{j, 0} \exp \left(T_{j}\left(r-\log (\delta)+\frac{s^{2}}{1-\alpha_{j}}\right)\right) .
\end{aligned}
$$


As long as this approximation holds for all $j$, the optimal solution to Problem (16) is to set $j^{\star}=\arg \max _{j=1, \ldots, J}\left\{T_{j}\left(r-\log (\delta)+\frac{s^{2}}{1-\alpha_{j}}\right)\right\}, w_{j^{\star}, 0}=1$ and $w_{j, 0}=0$ for all $j \neq j^{\star}$, that is, the investor only invests to reach goal $j^{\star}$. Inspecting the formula for $j^{\star}$, we see that $j^{\star}$ does not depend on the degrees of loss aversion (as long as these are moderate for all goals) and also not on the target wealths for the investment goals. Moreover, goal $j^{\star}$ is the one with the largest horizon when the parameters $\alpha_{j}$ (which determine the degrees of risk aversion/seeking) are similar among investment goals, and the logarithm of the discount rate $\delta$ is not extremely high relative to the risk-free rate of return $r$. By contrast, when $\alpha_{j}$ is significantly lower for long-term goals compared to short-term goals or the logarithm of the discount rate is extremely high compared to the risk-free rate of return, then the investor will only invest to reach short-term goals. ${ }^{5}$ These latter conditions do not seem to be realistic and we thus expect that in general goal $j^{\star}$ corresponds to the long-term goal. We come back to this in Sect. 3.

To conclude this subsection we report the full dynamics of optimal wealths and optimal strategies for all investment goals and at any time $t \in\left[0, T_{j}\right]$, as derived in Berkelaar [6, Proposition 5]:

Proposition 2.2 The optimal wealth $W_{j}(t)$ for investment goal $j$ and at time $t \in\left[0, T_{j}\right]$ corresponds to

$$
\begin{aligned}
& W_{j}^{\star}(t)=\bar{W}_{j} e^{-r\left(T_{j}-r\right)} \Phi\left(d_{1}\left(\xi^{\star}\left(y_{j}\right), T_{j}\right)\right) \\
& +\left(\frac{y_{j} \xi(t)}{\beta_{j}^{+} \alpha_{j}}\right)^{1 /\left(\alpha_{j}-1\right)} e^{\Gamma\left(t, T_{j}\right)} \Phi\left(d_{2}\left(\xi^{\star}\left(y_{j}\right), T_{j}\right)\right)
\end{aligned}
$$

where

$$
\begin{aligned}
d_{1}(\xi, T) & =\frac{\log (\xi / \xi(t))+\left(r-\frac{1}{2}\|k\|^{2}\right)(T-t)}{\|\kappa\| \sqrt{T-t}}, \\
d_{2}(\xi, T) & =d_{1}(\xi, T)+\|\kappa\| \frac{\sqrt{T-t}}{1-\alpha} \\
\Gamma(t, T) & =\frac{1-\alpha}{\alpha}\left(1+\frac{1}{2}\|\kappa\|^{2}\right)(T-t)+\frac{1}{2}\left(\frac{\alpha}{1-\alpha}\right)^{2}\|\kappa\|^{2}(T-t) .
\end{aligned}
$$

The optimal wealth share for goal $j$ at time $t \in\left[0, T_{j}\right]$ is given by

$$
w_{j}^{\star}(t)=\frac{W_{j}^{\star}(t)}{\sum_{j=1}^{J} W_{j}^{\star}(t)},
$$

and $w_{j}^{\star}(t)=0$ for $t>T_{j}$.

\footnotetext{
${ }^{5}$ For example, for $T_{1}=1$ and $T_{2}=5$ and $r=\log (\delta)$ one needs to have $\alpha_{2}<1-5\left(1-\alpha_{1}\right)$ to invest $100 \%$ of his wealth in the short-term goal 1 . On the other hand, when $\alpha_{1}=\alpha_{2}=\alpha$, one needs to have $\log (\delta)>r+s^{2} /(1-\alpha)$ in order to invest $100 \%$ of his wealth in the short-term goal 1 . With $\alpha=0.88$, as calibrated by Tversky and Kahneman [29] and $s=0.2$ this implies $\log (\delta)>r+0.3$.
} 
The optimal strategy $\lambda_{j}(t)=\left(\lambda_{j, 1}, \ldots, \lambda_{j, K}(t)\right)^{\prime}$ for investment goal $j$ at any time $t \in\left[0, T_{j}\right]$ corresponds to

$$
\begin{aligned}
\lambda_{j}(t) & =\frac{\left(\sigma^{\prime}\right)^{-1} \kappa}{W_{j}^{\star}(t)} \frac{\bar{W}_{j} e^{-r\left(T_{j}-t\right)} \varphi\left(d_{1}\left(\xi^{\star}\left(y_{j}\right), T_{j}\right)\right)}{\|\kappa\| \sqrt{\left(T_{j}-t\right)}} \\
+ & \frac{\left(\sigma^{\prime}\right)^{-1} \kappa}{W_{j}^{\star}(t)}\left(\left(\frac{y_{j} \xi(t)}{\beta_{j}^{+} \alpha_{j}}\right)^{1 /\left(\alpha_{j}-1\right)} e^{\Gamma\left(t, T_{j}\right)}\left(\frac{\varphi\left(d_{2}\left(\xi^{\star}\left(y_{j}\right), T_{j}\right)\right)}{\|\kappa\| \sqrt{\left(T_{j}-t\right)}}+\frac{\Phi\left(d_{2}\left(\xi^{\star}\left(y_{j}\right)\right), T_{j}\right)}{1-\alpha_{j}}\right)\right)
\end{aligned}
$$

where $\varphi=\Phi^{\prime}$ is the density function of the standard normal distribution.

\subsection{Optimal wealth shares with extreme loss aversion}

We now consider investors who display extreme loss aversion, that is, $\beta_{j}^{+}=0$ for all $j$. In this case Problem (16) is analytically tractable since we have an explicit expression for $h_{j}^{-1}$. As discussed before, extreme loss aversion describes investors who are mainly concerned about reaching their investment goals, while a surplus above their target wealth does not deliver any additional value to them. In our numerical exercises for the case $\beta_{j}^{+}>0$ (see Sect. 3), we found that all results for $\beta_{j}^{+}$between 0 and 0.1 are almost identical to the case $\beta_{j}^{+}=0$. Therefore, we interpret extreme loss aversion as when the degree of loss aversion $\beta_{j}^{-} / \beta_{j}^{+}$is higher than 10.

When $\beta_{j}^{+}=0$ for all $j$, we can prove the following proposition:

Proposition 2.3 Let $\beta_{j}^{+}=0$ for all $j$. Let $b_{j}=\bar{W}_{j} \exp \left(-r T_{j}\right) /\left(\xi_{0} W_{0}\right)$ and assume that $\sum_{j=1}^{J} b_{j} \geq 1$. Then

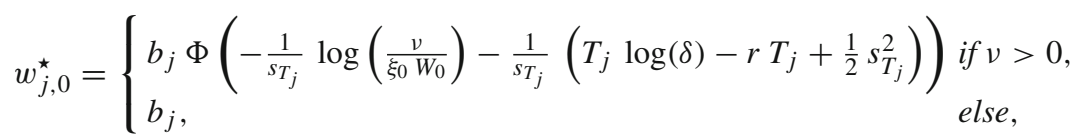

where $v$ solves $\sum_{j=1}^{J} w_{j, 0}^{\star}=1$.

The condition $\sum_{j=1}^{J} b_{j} \geq 1$ is equivalent to $\sum_{j=1} \bar{W}_{j} \exp \left(-r T_{j}\right) \geq W_{0}$, that is, the discounted value of all target wealths must be larger than or equal to the initial wealth. If the discounted value of all target wealths is strictly larger than the initial wealth $\left(\sum_{j=1}^{J} b_{j}>1\right)$ then we have $v>0$ and the investor invests some of her wealth into the risky assets. Therefore, in this case, the volatility of the market price of risk $s$ and the inter-temporal discount factor $\delta$ enter into the expression for wealth shares $w_{j, 0}^{\star}$. If $\sum_{j=1} \bar{W}_{j} \exp \left(-r T_{j}\right)=W_{0}$, then $v \leq 0$ and the investor can reach all investment goals with probability one by simply putting all her wealth into the risk-free asset. Therefore, in this case, $w_{j, 0}^{\star}$ simply corresponds to $b_{j}$, that is the ratio between the discounted value of the target wealth for goal $j$ and the initial wealth.

The ratio $b_{j}$ can be interpreted as a measures of how ambitious an investment goal is relative to the initial wealth. Obviously, we expect investors with extreme loss aversion to put a higher proportion of their wealth into goals with a higher discounted target wealth. Indeed, as we discussed above, if the initial wealth is high enough, then using the risk-free strategy will ensure that all investment goals will be reached with probability one, and investors put a higher proportion of their wealth into goals with higher $b_{j}$. However, when $\sum_{j=1}^{J} b_{j}>1$, 
the risk-free strategy causes investors to fails some of their investment goals with probability one. Therefore in this case investors might prefer having some risky assets into their portfolios and optimal wealth shares will then deviate from $b_{j}$. We also point out that when the risk-free strategy fails, then wealth shares $w_{j, 0}^{\star}$ are strictly smaller than $b_{j}$ for all investment goals. This means that investors decrease the proportion of wealth put into goal $j$ relative to $b_{j}$ for all investment goals, that is, instead of using the risk-free strategy for some goals and risky strategies for others, they prefer to invests into risky strategies for all investment goals. This is due to their risk-seeking behavior on losses.

The question now is how investors decide to split their wealth among investment goals when the risk-free strategy fails. In other words, on which goals do investors take more risk and put less wealth if we also account for how ambitious an investment goal is? In order to take into consideration the importance of one investment goal relative to the others, we consider the ratio $w_{j, 0}^{\star} / b_{j}$, which only depends on the characteristics of the market and the time horizon. We therefore analyze how the ratio $w_{j, 0}^{\star} / b_{j}$ changes as function of the time horizon. The results are reported in the following corollary, which directly follows from Proposition 2.3.:

Corollary 2.3 Let $v>0$ such that $\sum_{j=1}^{J} w_{j, 0}^{\star}=1$ and $w_{j, 0}^{\star}$ is given in Proposition 2.3. Then

$$
\frac{w_{j, 0}^{\star}}{b_{j}}=\Phi\left(-\frac{1}{s_{T_{j}}} \log \left(\frac{v}{\xi_{0} W_{0}}\right)-\frac{1}{s_{T_{j}}}\left(T_{j} \log (\delta)-r T_{j}+\frac{1}{2} s_{T_{j}}^{2}\right)\right)
$$

and the following holds:

(1) If $r-\log (\delta)-\frac{1}{2} s^{2}<0$, then the ratio $w_{j, 0} / b_{j}$ is maximal for

$$
T_{j}=\hat{T}_{j}=\frac{-\log \left(\frac{v}{\xi_{0} W_{0}}\right)}{r-\log (\delta)-\frac{1}{2} s^{2}},
$$

increasing for $T_{j}<\hat{T}_{j}$ and decreasing for $\hat{T}_{j}>T_{j}$ with $\lim _{T_{j} \rightarrow \infty} w_{j, 0}^{\star} / b_{j}=0$.

(2) If $r-\log (\delta)-\frac{1}{2} s^{2}>0$, then the ratio $w_{j, 0} / b_{j}$ is minimal for

$$
T_{j}=\hat{T}_{j}=\frac{-\log \left(\frac{v}{\xi_{0} W_{0}}\right)}{r-\log (\delta)-\frac{1}{2} s^{2}},
$$

strictly increasing for $T_{j}>\hat{T}_{j}$ with $\lim _{T_{j} \rightarrow \infty} w_{j, 0}^{\star} / b_{j}=1$, and strictly decreasing for $T_{j}<\hat{T}_{j}$.

(3) If $r-\log (\delta)-\frac{1}{2} s^{2}=0$, then the ratio $w_{j, 0} / b_{j}$ is strictly increasing for all $T_{j}$ if $\log \left(\nu /\left(\xi_{0} W_{0}\right)\right)>0$, strictly decreasing for all $T_{j}$ if $\log \left(v /\left(\xi_{0} W_{0}\right)\right)<0$ and constant if $\log \left(v /\left(\xi_{0} W_{0}\right)\right)=0$.

(4) Moreover,

$$
\lim _{T_{j} \rightarrow 0} \frac{w_{j, 0}^{\star}}{b_{j}}= \begin{cases}1, & \text { if } v<W_{0} \\ 0.5, & \text { if } v=W_{0} \\ 0, & \text { if } v>W_{0}\end{cases}
$$

We define the wealth ratio $W R_{0}=W_{0} / \sum_{j=1}^{J} \bar{W}_{j} \exp \left(-r T_{j}\right)$ as the ratio between the initial wealth and the discounted value of all target payoffs. Before we discuss Corollary 2.3, we briefly present here how $W R_{0}$ is linked to $v$. First, we notice that $1 / W R_{0}=\sum_{j=1}^{J} b_{j}$. 
Therefore, when $W R_{0}=1$ then also $\sum_{j=1}^{J} b_{j}=1$, and all investment goals can be reached with probability one by simply adopting the risk-free strategy for all goals. When $W R_{0}$ is smaller than 1 , then $\sum_{j=1}^{J} b_{j}$ is larger than 1 and optimal wealth shares differ from $b_{j}$. If $W R_{0}$ is very small, then $v$ must be very large in order to have $\sum_{j=1}^{J} w_{j, 0}^{\star}=1$. Indeed, $w_{j, 0}^{\star}$ strictly decreases with $v$ and a large $v$ is required in order to satisfies $\sum_{j=1}^{J} w_{j, 0}^{\star}=1$ when $\sum_{j=1}^{J} b_{j}$ is much larger than 1 . On the other hand, when $W R_{0}$ is slightly smaller than 1 , then $\sum_{j=1}^{J} b_{j}$ is slightly higher than 1 and a small $v>0$ is sufficient to have $\sum_{j=1}^{J} w_{j, 0}^{\star}=1$. Therefore, whether $v$ is large or small depends on how ambitious the investments goals are relative to the initial wealth, that is, on whether the wealth ratio $W R_{0}$ is very small or near to 1 . We now use this observation to discuss Corollary 2.3.

The quantity $r-\log (\delta)-(1 / 2) s^{2}$ can be written as $-\left[-\left(r+(1 / 2) s^{2}\right)+\log (\delta)+s^{2}\right]$, where $-\left(r+(1 / 2) s^{2}\right)$ is the growth rate of the pricing kernel, $s^{2}$ its volatility, and $\delta$ is the inter-temporal discount factor. Therefore, $r-\log (\delta)-(1 / 2) s^{2}$ is negative (positive), when the pricing kernel displays small (high) absolute growth rate, high (low) volatility, and, additionally, the inter-temporal discount factor is high (small). When these conditions hold, long-term investing appears less (more) attractive. The results in Corollary 2.3 are consistent with this observation, as will become clear from the following discussion.

Let us first consider the case $r-\log (\delta)-(1 / 2) s^{2}<0$, that is, long-term investing is less attractive. When the wealth ratio $W R_{0}$ is small enough such that $v$ is larger than $W_{0}$, then $\log \left(\nu /\left(\xi_{0} W_{0}\right)\right)$ is strictly positive and an intermediate horizon $\hat{T}_{j}$ exists where the corresponding ratio $w_{j, 0}^{\star} / b_{j}$ is maximal, while it decreases as the horizon increases. Note that $\hat{T}_{j}$ can be large when $W R_{0}$ is very small, that is, when the initial wealth is very small relative to the discounted sum of target wealths the ratio $w_{j, 0}^{\star} / b_{j}$ is maximal for a long-term goals. On the other hand, when $W R_{0}$ is slightly smaller than 1 such that $v$ is smaller than $W_{0}$, then $\log \left(\nu /\left(\xi_{0} W_{0}\right)\right)$ is strictly negative and the ratio $w_{j, 0}^{\star} / b_{j}$ strictly decreases with the time horizon. This means that in this case $w_{j, 0}^{\star} / b_{j}$ is maximal for very short-term goals, while it is small for long-term goals. Summarizing, when long-term investing is less attractive, investors put a higher proportion of their wealth (after accounting for how important the goal is) to long-term goals when the initial wealth is very small relative to the current value of target payoffs (goals are too ambitious), while they put a higher proportion of their wealth into short-term goals when goals are not too ambitious.

Let us now consider the case $r-\log (\delta)-(1 / 2) s^{2}>0$, that is, long-term investing is more attractive. If $W R_{0}$ is small enough such that $v>W_{0}$ and $\log \left(v /\left(\xi_{0} W_{0}\right)\right)$ is strictly positive, then $\hat{T}_{j}$ is negative, that is, the ratio $w_{j, 0}^{\star} / b_{j}$ strictly increases with $T_{j}$. It is therefore maximal for very long-term goals. On the other hand, when $W R_{0}$ is slightly smaller than 1 such that $v$ is smaller than $W_{0}$ and $\log \left(v /\left(\xi_{0} W_{0}\right)\right)$ is negative, then $\hat{T}_{j}$ is positive. Therefore, there exists an intermediate horizon $\hat{T}_{j}$ where the ratio $w_{j, 0}^{\star} / b_{j}$ is minimal, while it strictly increases for $T_{j}>\hat{T}_{j}$. Moreover, when $v<W_{0}$, the ratio is also maximal equals to 1 at $T_{j}=0$. Summarizing, when long-term investing is more attractive, the ratio $w_{j, 0}^{\star} / b_{j}$ is maximal for very long-term goals and, when goals are not too ambitious, also for very short-term investment goals. Table 1 summarizes the results in Corollary 2.3.

How does the investment strategy just discussed impact the probability of reaching the investment goals? The answer to this question follows directly from Proposition 2.3, Proposition 2.1 and Corollary 2.1, and is given in the following corollary:

Corollary 2.4 Let $v>0$ such that $\sum_{j=1}^{J} w_{j, 0}^{\star}=1$ and $w_{j, 0}^{\star}$ is given in Proposition 2.3. Then 
Table 1 Time horizon $T_{j}$ for which the ratio $w_{j, 0}^{\star} / b_{j}$ is maximal, as function of $\eta_{1}=r-\log (\delta)-(1 / 2) s^{2}$ and the wealth ratio $W R_{0}=W_{0} / \sum_{j=1}^{J} \bar{W}_{j} \exp \left(-r T_{j}\right)$. We have $\hat{T}_{j}=-\log \left(\nu /\left(\xi_{0} W_{0}\right)\right) / \eta_{1}$

\begin{tabular}{lll}
\hline & Wealth ratio $\left(W R_{0}\right)$ & High \\
\cline { 2 - 3 } & $\begin{array}{l}\text { Small } \\
\left(v>W_{0}\right)\end{array}$ & $\left(v<W_{0}\right)$ \\
\hline$\eta_{1}<0$ & $\hat{T}_{j}$ & 0 \\
$\eta_{1}>0$ & $\infty$ & 0 and $\infty$ \\
$\eta_{1}=0$ & $\infty$ & 0 \\
\hline
\end{tabular}

$$
P_{j}\left(T_{j}\right)=\mathbb{P}\left[W_{j}^{\star}\left(T_{j}\right) \geq \bar{W}_{j}\right]=\Phi\left(\frac{-\log \left(\frac{v}{\xi_{0} W_{0}}\right)-T_{j} \log (\delta)+r T_{j}+\frac{1}{2} s_{T_{j}}^{2}}{s_{T_{j}}}\right) .
$$

and the following holds:

(1) If $r-\log (\delta)+\frac{1}{2} s^{2}<0$, then $P_{j}\left(T_{j}\right)$ is maximal for

$$
T_{j}=\hat{T}_{j}=\frac{-\log \left(\frac{v}{\xi_{0} W_{0}}\right)}{r-\log (\delta)+\frac{1}{2} s^{2}},
$$

increasing for $T_{j}<\hat{T}_{j}$ and decreasing for $T_{j}>\hat{T}_{j}$, with $\lim _{T_{j} \rightarrow \infty} P_{j}\left(T_{j}\right)=0$.

(2) If $r-\log (\delta)+\frac{1}{2} s^{2}>0$, then $P_{j}\left(T_{j}\right)$ is minimal for

$$
T_{j}=\hat{T}_{j}=\frac{-\log \left(\frac{v}{\xi_{0} W_{0}}\right)}{r-\log (\delta)+\frac{1}{2} s^{2}},
$$

decreasing for $T_{j}<\hat{T}_{j}$ and increasing for $T_{j}>\hat{T}_{j}$ with $\lim _{T_{j} \rightarrow \infty} P_{j}\left(T_{j}\right)=1$.

(3) If $r-\log (\delta)+\frac{1}{2} s^{2}=0$, then $P_{j}\left(T_{j}\right)$ is constant as function of $T_{j}$.

(4) Moreover,

$$
\lim _{T_{j} \rightarrow 0} P_{j}\left(T_{j}\right)= \begin{cases}1, & \text { if } v<W_{0} \\ 0.5, & \text { if } v=W_{0} \\ 0, & \text { if } v>W_{0} .\end{cases}
$$

The quantity $r-\log (\delta)+(1 / 2) s^{2}$ can be written as $-\left[-\left(r+(1 / 2) s^{2}\right)+\log (\delta)\right]$. Thus $r-\log (\delta)+(1 / 2) s^{2}$ is negative (positive) when the absolute growth rate of the pricing kernel is small (high) and the inter-temporal discount factor is high (small). If the absolute growth rate of the pricing kernel is small, bad states of the world are more likely to occur. Therefore, the probability to reach an investment goal decreases, especially for long-term goals. On the other hand, if the absolute growth rate of the pricing kernel is large, good states of the world are more likely to occur and the probability to reach an investment goal is higher, especially for long-term goals. Finally, we also see that when the initial wealth is high enough, (very) short-term goals will be reached almost surely. This is due to the fact that for very short horizons wealth shares almost corresponds to $b_{j}$ (the risk-free strategy), as reported in Corollary 2.3. Table 2 summarizes these findings.

We conclude this section with a brief discussion on whether goal-based investing is inefficient from the perspective of an investor who add up the different investment goals and 
Table 2 Time horizon $T_{j}$ for which the probability to reach the investment goal is maximal, as function of $\eta_{2}=r-\log (\delta)+(1 / 2) s^{2}$ and the wealth ratio $W R_{0}=W_{0} / \sum_{j=1}^{J} \bar{W}_{j} \exp \left(-r T_{j}\right)$. We have $\hat{T}_{j}=$ $-\log \left(v /\left(\xi_{0} W_{0}\right)\right) / \eta_{2}$

\begin{tabular}{lll}
\hline & Wealth ratio $\left(W R_{0}\right)$ & High \\
& $\begin{array}{l}\text { Small } \\
\left(\nu>W_{0}\right)\end{array}$ & $\left(v<W_{0}\right)$ \\
\hline$\eta_{2}<0$ & $\hat{T}_{j}$ & 0 \\
$\eta_{2}>0$ & $\infty$ & 0 and $\infty$ \\
$\eta_{2}=0$ & $\infty$ & 0 \\
\hline
\end{tabular}

derive utility from total wealth. We restrict ourselves to the case where all investment goals have the same time horizon, as in Das et al. [12]. In this case we find that goal-based investing delivers the same optimal terminal total wealth (and investment strategy) as when investors consider a unique goal with target wealth equals to the sum of goals' specific target wealths. This result is reported in the following corollary.

Corollary 2.5 Let $\beta_{j}^{+}=0$ for all $j$ and $T_{j}=T$ for all $j$. Then the optimal terminal total wealth is

$$
W^{\star}(T)=\left\{\begin{array}{cc}
\sum_{j=1}^{J} \bar{W}_{j}, & \text { if } \xi(T)<\xi^{\star}(y) \\
0, & \text { if } \xi(T) \geq \xi^{\star}(y)
\end{array}\right.
$$

where $\xi^{\star}(y)=\xi^{\star}\left(y_{j}\right)$ is independent from $j$. This corresponds to the terminal wealth for a unique investment goal with time horizon $T$ and target wealth $\sum_{j=1}^{J} \bar{W}_{j}$.

Corollary 2.5 implies that, when all investment goals possess the same horizon, goal-based investing is not inefficient from the perspective of extremely loss averse investors who add up the different investment goals and derive utility from total wealth. This result is similar to that reported by Das et al. [12] for the case of goal-based investing with mean-variance preferences.

\section{Numerical examples}

To illustrate the implications of the model presented in the previous section, we derive the optimal investment strategy for an investor with preferences according to Eq. 4 and multiple investment goals at different horizons.

We assume that the investor has three investment goals at different time horizons. We assume that $\bar{W}_{j}=\$ 50,000 \exp \left(r T_{j}\right)$ for $j=1,2,3$ where $T_{1}=1$ year (short-term), $T_{2}=5$ years (medium term) and $T_{3}=20$ years (long-term), that is, all investment goals have the same discounted value equal to $\$ 50,000$. Under this assumption, the parameters $b_{j}$ from Proposition 2.3 are identical for all investment goals and thus wealth shares are not affected by the how ambitious an investment goal is relative to the others.

We specify the investor's preferences assuming $\beta_{j}^{-}=2.25$ and $\alpha_{j}=0.88$ for all $j=$ $1,2,3$ and $\delta=\exp (r)$, where $r$ is the risk-free rate of return, while $\beta_{j}^{+}$is not fixed and will determine the degree of loss aversion $\beta_{j}=\beta_{j}^{-} / \beta_{j}^{+}$. In our numerical examples we further assume that there is one risky asset, that is the market portfolio, with drift $\mu$ and volatility $\sigma$. The Sharpe ratio corresponds to $\kappa=(\mu-r) / \sigma$. 

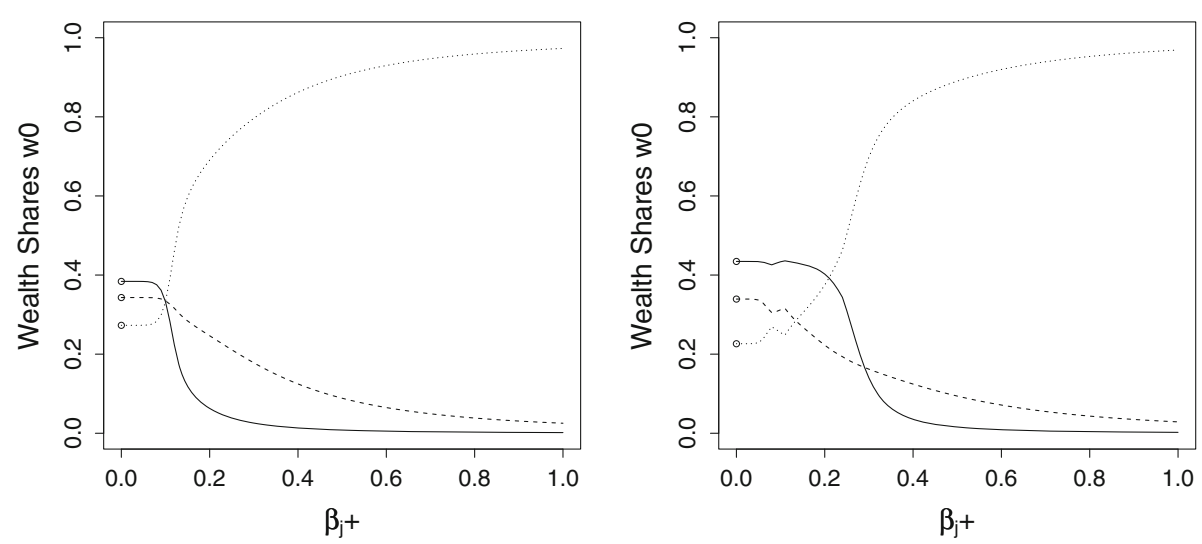

Fig. 2 Optimal wealth shares at time $t=0$ as function $\beta_{j}^{+}$(loss aversion parameter), when the investor has three investment goals with same discounted value at time $t=0$ (that is, $\bar{W}_{j} \exp \left(-r T_{j}\right)=\$ 50,000$ for all $j$ ) at horizons 1 year (full line), 5 years (dashed line) and 20 years (dotted line). Investor's preferences are characterized by $\beta_{j}^{-}=2.25$, and $\alpha_{j}=0.88$ for $j=1,2,3$. We have $r=0.03, \sigma=0.2$ and $\kappa=0.2$, which imply an equity premium of $4 \%$. The wealth ratio $W R_{0}=W_{0} / \sum_{j=1}^{3} \bar{W}_{j} \exp \left(-r T_{j}\right)$ corresponds to 0.4 (left panel) and 0.75 (right panel)

When $\beta_{j}^{+} \neq 0$ and the degree of loss aversion $\beta_{j}=\beta_{j}^{-} / \beta_{j}^{+}$is in the range of two-three for all $j$, as calibrated by Tversky and Kahneman [29], we see that the investor optimally puts almost all her wealth in the long-term goal. This happens also if the degree of loss aversion is much higher for the short-term goal than for the long-term goal. This result in now clear from our discussion in Sect. 2, where we showed that for moderate loss aversion the investor puts all her wealth in a unique goal and this corresponds to the long-term goal under realistic assumptions for the preference parameters. Figure 2 displays optimal wealth shares as function of $\beta_{j}^{+}$, under the assumption that $\beta_{j}^{-}=2.25$ for all $j$. For $\beta_{j}^{+}$in the range from 0.6 to 1 , which implies a degree of loss aversion from 2.25 to 3.75 , almost all wealth is invested in the long-term goal. This result is robust with respect to different values for the wealth ratio ( 0.4 in the left panel and 0.75 in the right panel). It follows that in case that the degree of loss aversion is in the range two-three, the overall investment strategy almost corresponds to the long-term investment strategy, as shown in Fig. 3.

We now discuss the case with $\beta_{j}^{+}=0$ for all $j$, that is, the investor displays extreme loss aversion. As we see from Fig. 2, wealth shares are almost constant as function of $\beta_{j}^{+}$ for $\beta_{j}^{+} \in(0,0.1)$. We found this in all our numerical exercises. Thus $\beta_{j}^{+}=0$ can be seen as a good approximation for the case $\beta_{-}^{j} / \beta_{j}^{+}>10$, that is, the degree of loss aversion is higher than 10. Figure 4 shows optimal wealth shares at time 0 as function of the wealth ratio $W R_{0}=W_{0} / \sum_{j=1}^{3} \bar{W}_{j} \exp \left(-r T_{j}\right)$. We assumed $r=0.03, \sigma=0.2$, while $\kappa$ takes values 0.2 (left panel) and 0.4 (right panel), which imply an equity premium of $4 \%$ and $8 \%$, respectively. With $r=0.03$ we have $\bar{W}_{1}=\$ 51,523, \bar{W}_{2}=\$ 58,092$ and $\bar{W}_{3}=\$ 91,106$. When the wealth ratio is small (that is, goals are too ambitious), the investor puts the largest proportion of her wealth to the long-term goal. However, as the wealth ratio increases (that is, goals are less ambitious) the investor mainly invests to reach short-term goals, that is, the probability to reach this goal is significantly higher, as shown in Fig. 5. We also see that when the wealth ratio is small, the probability to reach the long-term goal is higher. By contrast, in this case, the probability to reach the short-term goal is small, and the investor adopts 

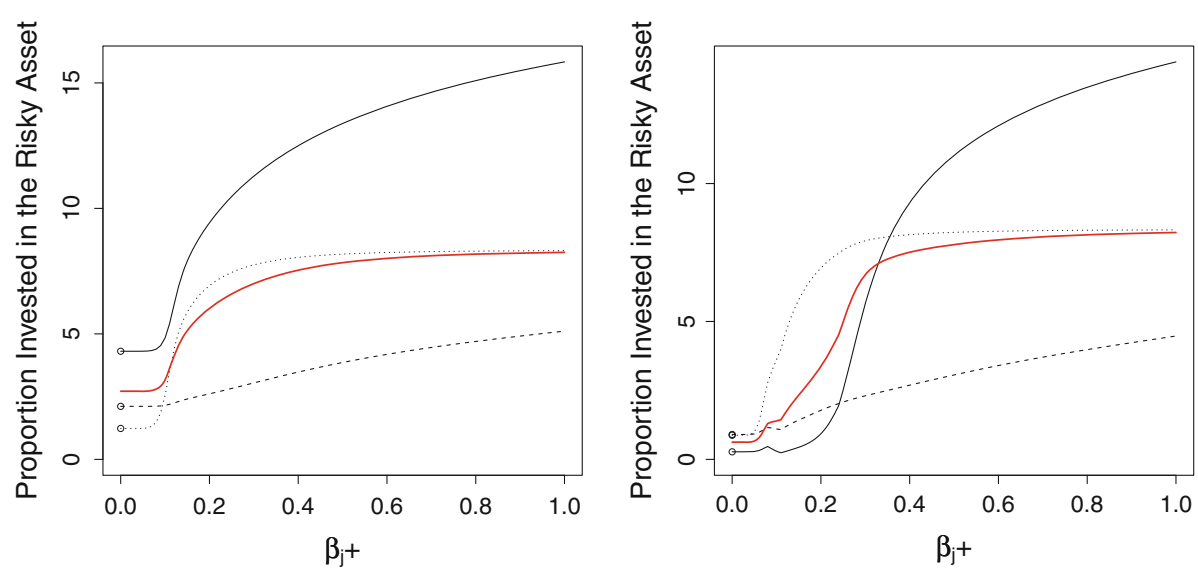

Fig. 3 Optimal proportion of risky assets at time $t=0$ as function $\beta_{j}^{+}$(loss aversion parameter), when the investor has three investment goals with same discounted value at time $t=0$ (that is, $\bar{W}_{j} \exp \left(-r T_{j}\right)=$ $\$ 50,000$ for all $j$ ) at horizons 1 year (full line), 5 years (dashed line) and 20 years (dotted line). The red line gives the total allocation to the risky asset. Investor's preferences are characterized by $\beta_{j}^{-}=2.25$, and $\alpha_{j}=0.88$ for $j=1,2,3$. We have $r=0.03, \sigma=0.2$ and $\kappa=0.2$, which imply an equity premium of $4 \%$. The wealth ratio $W R_{0}=W_{0} / \sum_{j=1}^{3} \bar{W}_{j} \exp \left(-r T_{j}\right)$ corresponds to 0.4 (left panel) and 0.75 (right panel)
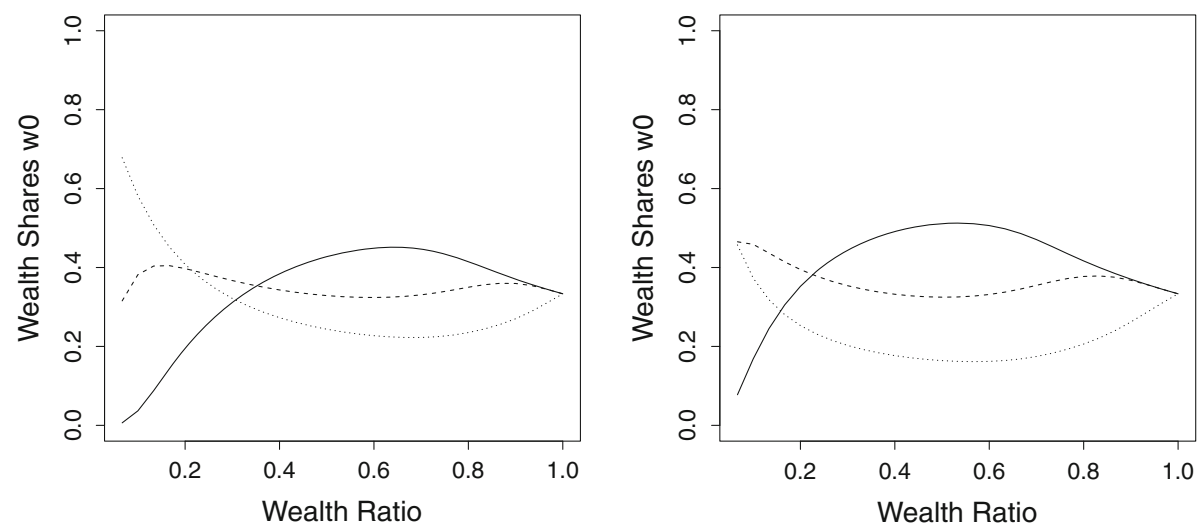

Fig. 4 Optimal wealth shares at time $t=0$ as function of the of the wealth ratio $W R_{0}=$ $W_{0} / \sum_{j=1}^{3} \bar{W}_{j} \exp \left(-r T_{j}\right)$, when the investor has three investment goals with same discounted value at time $t=0$ (that is, $\bar{W}_{j} \exp \left(-r T_{j}\right)=\$ 50,000$ for all $j$ ) at horizons 1-year (full line), 5 years (dashed line) and 20 years (dotted line). Investor's preferences are characterized by $\beta_{j}^{+}=0, \beta_{j}^{-}=2.25$ (extreme loss aversion) and $\alpha_{j}=0.88$ for $j=1,2,3$. We have $r=0.03, \sigma=0.2$. We have $\kappa=0.2$ (left panel) and $\kappa=0.4$ (right panel), which imply an equity premium of $4 \%$ and $8 \%$, respectively

aggressive investment strategies for all investment goals, as shown in Fig. 6. Moreover, short- and medium-term goals displays a high leverage and this is true also for the overall investment strategy. We also note that these results do not qualitatively depend on the equity premium.

Figure 7 shows the optimal wealth shares as function of the equity premium. We fixed the volatility $\sigma=0.20$ and the wealth ratio takes values 0.40 (left panel) and 0.75 (right panel), which correspond to $W_{0}=\$ 60,000$ and $W_{0}=\$ 112,500$, respectively (the discounted 

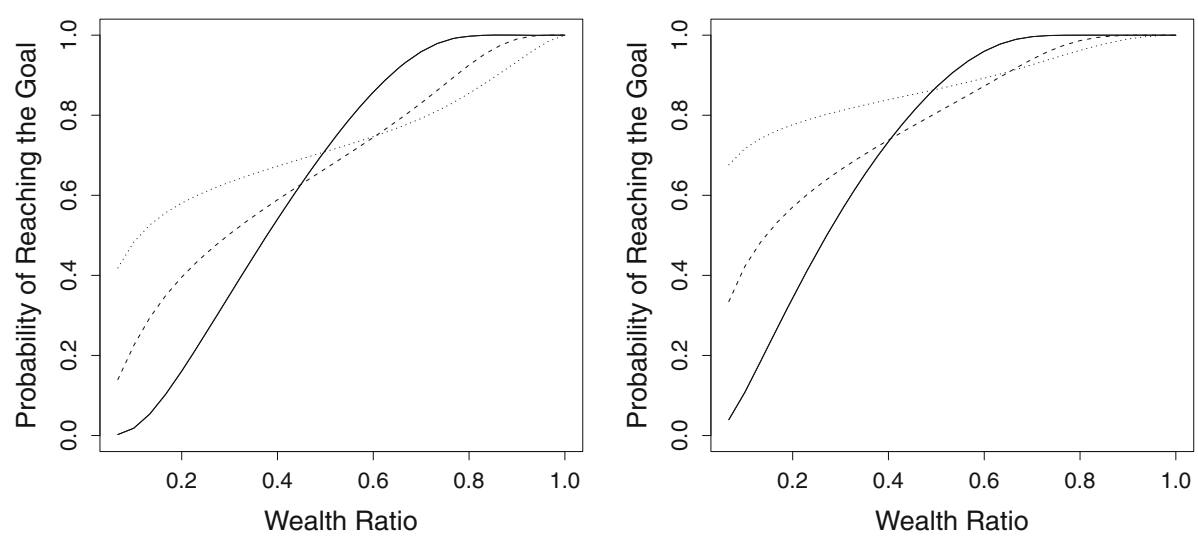

Fig. 5 Probability of reaching the investment goals as function of the of the wealth ratio $W R_{0}=$ $W_{0} / \sum_{j=1}^{3} \bar{W}_{j} \exp \left(-r T_{j}\right)$, when the investor has three investment goals with same discounted value at time $t=0$ (that is, $\bar{W}_{j} \exp \left(-r T_{j}\right)=\$ 50,000$ for all $j$ ) at horizons 1-year (full line), 5 years (dashed line) and 20 years (dotted line). Investor's preferences are characterized by $\beta_{j}^{+}=0, \beta_{j}^{-}=2.25$ (extreme loss aversion) and $\alpha_{j}=0.88$ for $j=1,2,3$. We have $r=0.03, \sigma=0.2$. We have $\kappa=0.2$ (left panel) and $\kappa=0.4$ (right panel), which imply an equity premium of $4 \%$ and $8 \%$, respectively
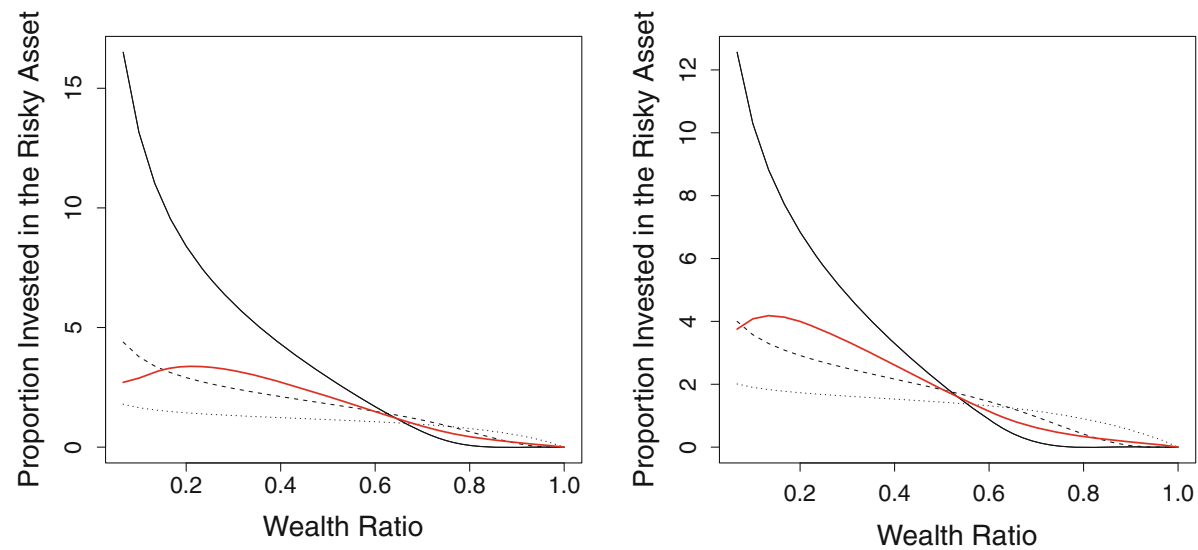

Fig. 6 Optimal proportion of risky assets at time $t=0$ as function of the wealth ratio $W R_{0}=$ $W_{0} / \sum_{j=1}^{3} \bar{W}_{j} \exp \left(-r T_{j}\right)$, when the investor has three investment goals with same discounted value at time $t=0$ (that is, $\bar{W}_{j} \exp \left(-r T_{j}\right)=\$ 50,000$ for all $j$ ) at horizons 1 year (full line), 5 years (dashed line) and 20 years (dotted line). The red line gives the total allocation to the risky asset. Investor's preferences are characterized by $\beta_{j}^{+}=0, \beta_{j}^{-}=2.25$ (extreme loss aversion) and $\alpha_{j}=0.88$ for $j=1,2,3$. We have $r=0.03, \sigma=0.2$. We have $\kappa=0.2$ (left panel) and $\kappa=0.4$ (right panel), which imply an equity premium of $4 \%$ and $8 \%$, respectively

value of all target payoffs is $\$ 150,000$ ). We see that investors put a higher proportion of initial wealth into short-term goals as the equity premium increases. Nevertheless, as show in Fig. 8, the probability to reach the short-term goal is the lowest when the wealth ratio is low. By contrast, it is the highest when the wealth ratio is high. Figure 9 reports the investment strategies for all goals and globally. When the wealth ratio is low, the shortterm strategy becomes less aggressive as the equity premium increases, while the opposite holds for the long-term strategy. However, in this case, the overall investment strategy is 

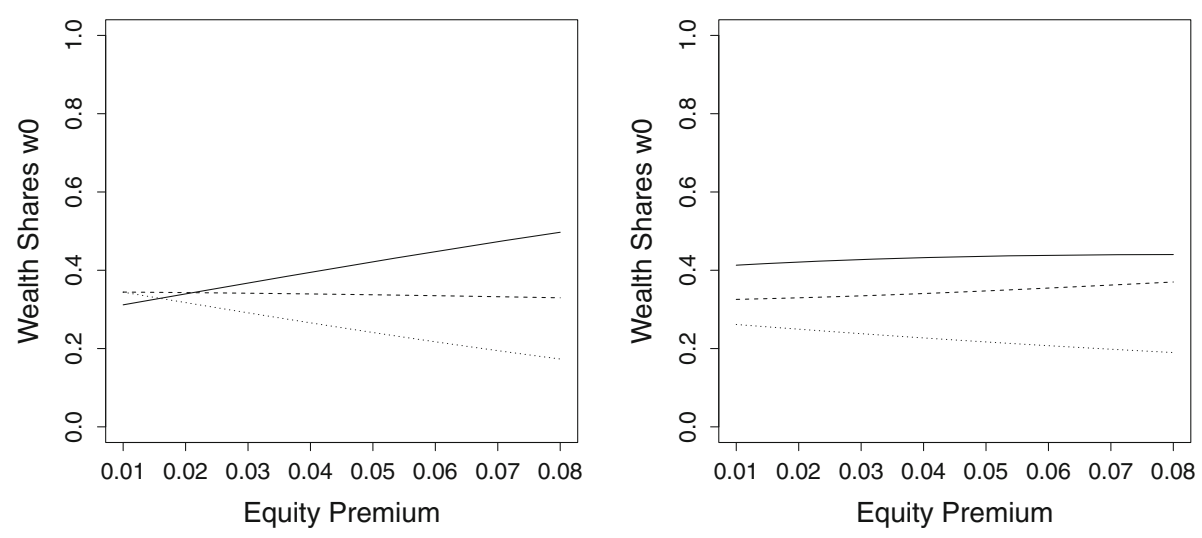

Fig. 7 Optimal wealth shares at time $t=0$ as function of the equity premium $\mu-r=\sigma \kappa$, when the investor has three investment goals with same discounted value at time $t=0$ (that is, $\bar{W}_{j} \exp \left(-r T_{j}\right)=\$ 50,000$ for all $j$ ) at horizons 1 year (full line), 5 years (dashed line) and 20 years (dotted line). Investor's preferences are characterized by $\beta_{j}^{+}=0, \beta_{j}^{-}=2.25$ (extreme loss aversion) and $\alpha_{j}=0.88$ for $j=1,2,3$. We have $r=0.03, \sigma=0.2$. The wealth ratio $W R_{0}=W_{0} / \sum_{j=1}^{3} \bar{W}_{j} \exp \left(-r T_{j}\right)$ corresponds to 0.40 (left panel) and 0.75 (right panel)
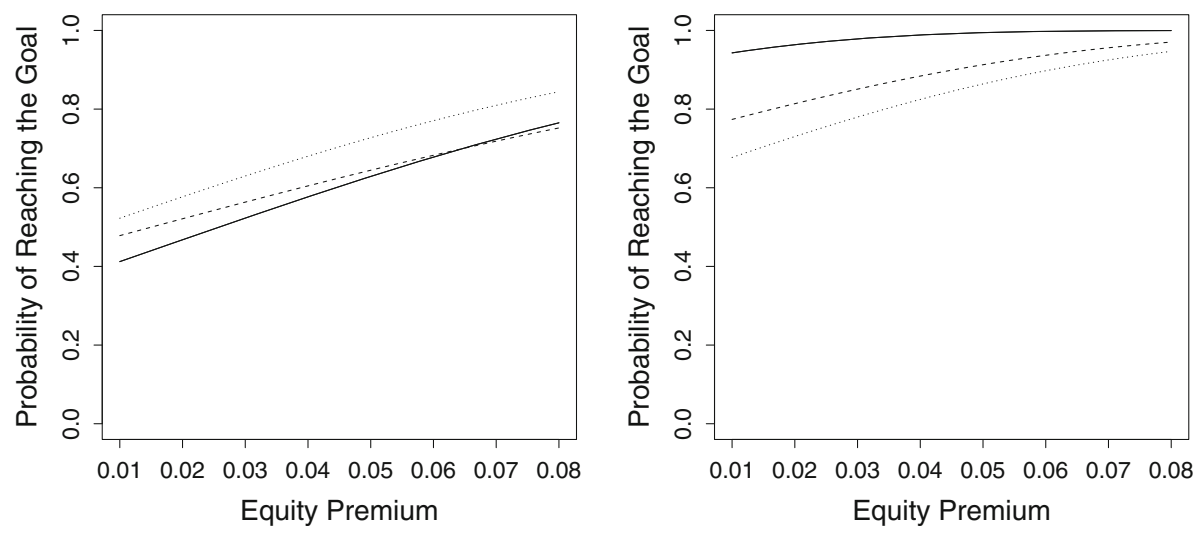

Fig. 8 Probability of reaching the investment goals as function of the equity premium $\mu-r=\sigma \kappa$, when the investor has three investment goals with same discounted value at time $t=0$ (that is, $\bar{W}_{j} \exp \left(-r T_{j}\right)=$ $\$ 50,000$ for all $j$ ) at horizons 1 year (full line), 5 years (dashed line) and 20 years (dotted line). Investor's preferences are characterized by $\beta_{j}^{+}=0, \beta_{j}^{-}=2.25$ (extreme loss aversion) and $\alpha_{j}=0.88$ for $j=1,2,3$. We have $r=0.03, \sigma=0.2$. The wealth ratio $W R_{0}=W_{0} / \sum_{j=1}^{3} \bar{W}_{j} \exp \left(-r T_{j}\right)$ corresponds to 0.40 (left panel) and 0.75 (right panel)

almost unaffected by the equity premium. When the wealth ratio is high, goals' specific strategies displays similar patterns as when the wealth ratio is low. However, the overall investment strategy now becomes less aggressive as the equity premium increases. This is because in this case the long-term goal can be reached with higher probability and extremely loss averse investors allocate less wealth to it. Consequently, they allocate more wealth to short- and medium-term goals and adopt safer strategies for those goals. Finally, in this case, the impact of short- and medium-term strategies on the overall investment strategy is 

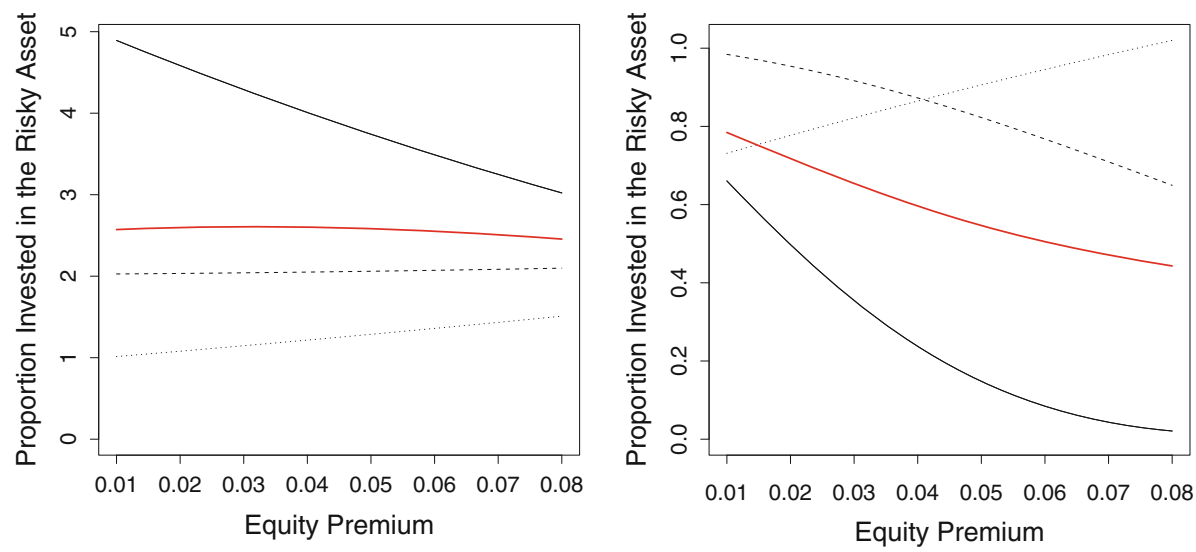

Fig. 9 Optimal proportion of risky assets at time 0 as function of the equity premium $\mu-r=\sigma \kappa$, when the investor has three investment goals with same discounted value at time $t=0$ (that is, $\bar{W}_{j} \exp \left(-r T_{j}\right)=$ $\$ 50,000$ for all $j$ ) at horizons 1 year (full line), 5 years (dashed line) and 20 years (dotted line). The red line gives the total allocation to the risky asset. Investor's preferences are characterized by $\beta_{j}^{+}=0, \beta_{j}^{-}=2.25$ (extreme loss aversion) and $\alpha_{j}=0.88$ for $j=1,2,3$. We have $r=0.03, \sigma=0.2$. The wealth ratio $W R_{0}=W_{0} / \sum_{j=1}^{3} \bar{W}_{j} \exp \left(-r T_{j}\right)$ corresponds to 0.40 (left panel) and 0.75 (right panel)

stronger and thus the overall investment strategy also becomes safer as the equity premium increases.

\section{Conclusion}

In this paper we studied loss aversion in a portfolio selection model, where investors possess different investment goals at different time horizons. Our model assumes that investors mentally organize each investment goal as a separate account and derive optimal investment strategies for each investment goal, ignoring covariances between goal-specific portfolios. Nevertheless, they initially have to decide how to split wealth among investment goals. Therefore, goals are at least partially connected, that is, our model only assumes partial narrow framing.

We numerically derived optimal wealth shares allocated to each investment goal and optimal investment strategies for all goals. For the case of extreme loss aversion we also derived an analytical expression for wealth shares. We showed that loss averse investors mainly invest too reach long-term goals and adopt aggressive investment strategies for short-term goals. The same behavior follows when loss aversion is extremely high and investment goals are very ambitious. By contrast, when goals are not too ambitious, extremely loss averse investors display a short-term perspective and the probability to reach short-term goals is higher compared to medium- and long-term goals.

Our model implies excessive risk taking when investors possess ambitious (short-term) goals. Indeed, when goals are very ambitious, the likelihood that investors will face losses is higher. Since investors are assumed to be risk seeking on losses, when goals become more ambitious, they are willing to take more risk. This formalizes a remark made by Shefrin [26], who argues that ambitious short-term goals have generated a high level of leverage, and this has been identified as one of the main drivers of the financial crises erupted in 2008 . 
Acknowledgments Financial support from the Swiss National Science Foundation (grant 105214-130078), the National Center of Competence in Research "Financial Valuation and Risk Management" (NCCR-FINRISK) and the Foundation for Research and Development of the University of Lugano is gratefully acknowledged. The author thank the editors and one referee for their useful comments. The author also benefited from discussions with David Brown, Alessandra Cillo, Thorsten Hens, Thierry Post, Melvyn Sim and Hersh Shefrin.

\section{A Proofs}

\section{A.1 Proof of Proposition 2.1}

See Berkelaar et al. [6]. We additionally point out that $g_{j}(\cdot, y)$ is a continuous, strictly decreasing function. For $y>0$ fix, we have $\lim _{x \rightarrow 0} g_{j}(x, y)=\infty$ and $\lim _{x \rightarrow \infty} g_{j}(x, y)=-\infty$. So for each $y>0$ we find a unique $x$ such that $g_{j}(x, y)=0$. For $y>0$ fix, we denote by $\xi_{j}^{\star}(y)$ the solution to $g_{j}(x, y)=0$.

\section{A.2 Proof of Lemma 2.1}

Assume that $\xi^{\star}(y)=a / y^{b}$ for some $a, b \in \mathbb{R}$. We have:

$$
\begin{aligned}
g\left(\xi^{\star}(y), y\right) & =\frac{1-\alpha}{\alpha}\left(\frac{y^{b}}{a x}\right)^{\alpha /(1-\alpha)}\left(\beta^{+} \alpha\right)^{1 /(1-\alpha)}-\bar{W} y a y^{-b}+\beta^{-} \bar{W}^{\alpha} \\
& =\frac{1-\alpha}{\alpha} a^{-\alpha /(1-\alpha)} y^{(b-1) \alpha /(1-\alpha)}\left(\beta^{+} \alpha\right)^{1 /(1-\alpha)}-a \bar{W} y^{-b+1}+\beta^{-} \bar{W}^{\alpha} .
\end{aligned}
$$

This expression is constant if and only if $b=1$. In this case we have:

$$
\left.g\left(\xi^{\star}(y), y\right)\right)=\frac{1-\alpha}{\alpha} a^{-\alpha /(1-\alpha)}\left(\beta^{+} \alpha\right)^{1 /(1-\alpha)}-a \bar{W}+\beta^{-} \bar{W}^{\alpha}
$$

and thus $\left.g\left(\xi^{\star}(y), y\right)\right)=0$ if and only if

$$
\frac{1-\alpha}{\alpha} a^{-\alpha /(1-\alpha)}\left(\beta^{+} \alpha\right)^{1 /(1-\alpha)}-a \bar{W}+\beta^{-} \bar{W}^{\alpha}=0 .
$$

We still have to show that $a>0$ and is unique. Note that

$$
\frac{1-\alpha}{\alpha} a^{-\alpha /(1-\alpha)}\left(\beta^{+} \alpha\right)^{1 /(1-\alpha)}-a \bar{W}+\beta^{-} \bar{W}^{\alpha}=g(a, 1)
$$

and $g(\cdot, 1)$ is strictly decreasing, $\lim _{a \rightarrow 0} g(a, 1)=\infty$ and $\lim _{a \rightarrow \infty} g(a, 1)=-\infty$. So, $g(a, 1)=0$ possesses a unique solution $a>0$ and the statement in the Lemma follows.

\section{A.3 Proof of Lemma 2.2}

From Proposition 2.1, it follows:

$$
\begin{aligned}
\mathbb{E}\left[\xi(T) W^{\star}(T)\right] & =\mathbb{E}\left[\bar{W} \xi(T) 1_{\xi(T) \leq \xi^{\star}(y)}+\left(\frac{y}{\beta^{+} \alpha}\right)^{\frac{1}{\alpha-1}} \xi(T)^{\frac{\alpha}{\alpha-1}} 1_{\xi(T) \leq \xi^{\star}(y)}\right] \\
& =\bar{W} \mathbb{E}\left[\xi(T) 1_{\xi(T) \leq \xi^{\star}(y)}\right]+\left(\frac{y}{\beta^{+\alpha}}\right)^{\frac{1}{\alpha-1}} \mathbb{E}\left[\xi(T)^{\frac{\alpha}{\alpha-1}} 1_{\xi(T) \leq \xi^{\star}(y)}\right] .
\end{aligned}
$$


Since $\xi(T)$ is log-normally distributed with parameters $m_{T}=m T$ and $s_{T}=s \sqrt{T}$, then

$$
\mathbb{E}\left[\xi(T) 1_{\left.\xi(T) \leq \xi^{\star}(y)\right]}=\exp \left(m_{T}+\frac{1}{2} s_{T}^{2}\right) \Phi\left(\frac{\log \left(\xi^{\star}(y)\right)-m_{T}-s_{T}^{2}}{s_{T}}\right) .\right.
$$

Moreover, $\xi(T)^{\alpha /(\alpha-1)}$ is also log-normally distributed with parameters $\alpha m_{T} /(\alpha-1)$ and $\alpha s_{T} /(1-\alpha)$. It follows

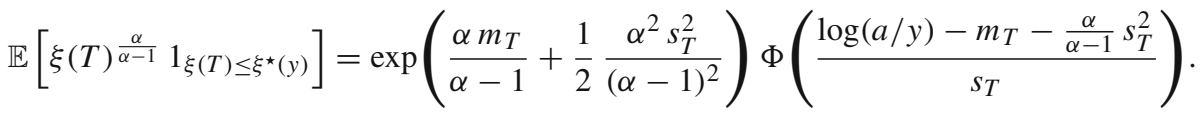

Let $b=\frac{\bar{W}}{\xi_{0} W_{0}} \exp \left(m_{T}+\frac{1}{2} s_{T}^{2}\right)$

$=\frac{\bar{W}}{\xi_{0} W_{0}} \exp (-r T)$ and $c=\frac{1}{\xi_{0} W_{0}}\left(\beta^{+} \alpha\right)^{\frac{1}{1-\alpha}} \exp \left(\frac{\alpha m_{T}}{\alpha-1}+\frac{1}{2} \frac{\alpha^{2} s_{T}^{2}}{(\alpha-1)^{2}}\right)$ then

$$
\begin{aligned}
& \frac{\mathbb{E}\left[\xi(T) W^{\star}(T)\right]}{\xi_{0} W_{0}} \\
& =b \Phi\left(\frac{\log \left(\xi^{\star}(y)\right)-m_{T}-s_{T}^{2}}{s_{T}}\right)+c y^{\frac{1}{\alpha-1}} \Phi\left(\frac{\log (a / y)-m_{T}-\frac{\alpha}{\alpha-1} s_{T}^{2}}{s_{T}}\right) .
\end{aligned}
$$

Now let

$$
h(y)=b \Phi\left(\frac{\log \left(\xi^{\star}(y)\right)-m_{T}-s_{T}^{2}}{s_{T}}\right)+c y^{\frac{1}{\alpha-1}} \Phi\left(\frac{\frac{\log (a / y)-m_{T}-\frac{\alpha}{\alpha-1} s_{T}^{2}}{1-\alpha}}{s_{T}}\right)
$$

then

$$
\mathbb{E}\left[\xi(T) W^{\star}(T)\right]=\xi_{0} w_{0} W_{0} \Leftrightarrow h(y)=w_{0} .
$$

Putting $\xi^{\star}(y)=a / y$ from Lemma 2.1, the statement in the Lemma follows.

\section{A.4 Proof of Corollary 2.2}

Let $a$ and $y$ be solutions to Eq. 12 and $h(y)=w_{0}$, respectively, given parameters $\beta^{+}$and $\beta^{-}$. Let $\tilde{\beta}^{+}=u \beta^{+}$and $\tilde{\beta}^{-}=u \beta^{-}$, while the other parameters are fix. Denote by $\tilde{a}$ and $\tilde{y}$ the solutions to Eq. 12 and $h(y)=w_{0}$, respectively, given parameters $\tilde{\beta}^{+}$and $\tilde{\beta}^{-}$. It can be easily shown that $\tilde{a}=u a$ and $\tilde{y}=u y$. Therefore, $\xi^{\star}(\tilde{y})=\tilde{a} / \tilde{y}=a / y=\xi^{\star}(y)$. Moreover, since the surplus in the good scenario depends on the ratio $y / \beta^{+}$, it is also independent from $u$.

\section{A.5 Proof of Lemma 2.3}

From Proposition 2.1, it follows:

$$
\begin{aligned}
\mathbb{E}\left[W^{\star}(T)\right] & =\mathbb{E}\left[\bar{W} 1_{\xi(T) \leq \xi^{\star}(y)}+\left(\frac{y}{\beta^{+\alpha}}\right)^{\frac{1}{\alpha-1}} \xi(T)^{\frac{1}{\alpha-1}} 1_{\xi(T) \leq \xi^{\star}(y)}\right] \\
& =\bar{W} \mathbb{P}\left[\xi(T) \leq \xi^{\star}(y)\right]+\left(\frac{y}{\beta^{+\alpha}}\right)^{\frac{1}{\alpha-1}} \mathbb{E}\left[\xi(T)^{\frac{1}{\alpha-1}} 1_{\xi(T) \leq \xi^{\star}(y)}\right] .
\end{aligned}
$$


Since $\xi(T)$ is log-normally distributed with parameters $m_{T}=m T$ and $s_{T}=s \sqrt{T}$, then

$$
\mathbb{P}\left[\xi(T) \leq \xi^{\star}(y)\right]=\Phi\left(\frac{\log \left(\xi^{\star}(y)\right)-m_{T}}{s_{T}}\right) .
$$

Moreover, $\xi(T)^{1 /(\alpha-1)}$ is also log-normally distributed with parameters $m_{T} /(\alpha-1)$ and $s_{T} /(1-\alpha)$. It follows

$$
\mathbb{E}\left[\xi(T)^{\frac{1}{\alpha-1}} 1_{\xi(T) \leq \xi^{\star}(y)}\right]=\exp \left(\frac{m_{T}}{\alpha-1}+\frac{1}{2} \frac{s_{T}^{2}}{(\alpha-1)^{2}}\right) \Phi\left(\frac{\log \left(\xi^{\star}(y)\right)-m_{T}+\frac{s_{T}^{2}}{1-\alpha}}{s_{T}}\right) .
$$

Let $d=\left(\beta^{+} \alpha\right)^{\frac{1}{1-\alpha}} \exp \left(\frac{m_{T}}{\alpha-1}+\frac{1}{2} \frac{s_{T}^{2}}{(\alpha-1)^{2}}\right)$ then

$$
\mathbb{E}\left[W^{\star}(T)\right]=\bar{W} \Phi\left(\frac{\log \left(\xi^{\star}(y)\right)-m_{T}}{s_{T}}\right)+d y^{\frac{1}{\alpha-1}} \Phi\left(\frac{\log \left(\xi^{\star}(y)\right)-m_{T}+\frac{s_{T}^{2}}{1-\alpha}}{s_{T}}\right) .
$$

We define

$$
k(y)=\bar{W} \Phi\left(\frac{\log \left(\xi^{\star}(y)\right)-m_{T}}{s_{T}}\right)+d y^{\frac{1}{\alpha-1}} \Phi\left(\frac{(1-\alpha) \log \left(\xi^{\star}(y)\right)+m_{T}-\frac{s_{T}^{2}}{1-\alpha}}{s_{T}}\right) .
$$

\section{A.6 Proof of Proposition 2.3}

When $\beta_{j}^{+}=0$, we know from Corollary 2.1 that

$y_{j}=a_{j} \exp \left(-s_{T_{j}} \Phi^{-1}\left(w_{j, 0} / b_{j}\right)-m_{T_{j}}-s_{T_{j}}^{2}\right)$ solves $h_{j}(y)=w_{j, 0}$. We put $y_{j}$ into $k_{j}$ and obtain

$$
f_{j}\left(w_{0, j}\right)=k_{j}\left(h_{j}^{-1}\left(w_{j, 0}\right)\right)=\bar{W}_{j} \Phi\left(\Phi^{-1}\left(w_{j, 0} / b_{j}\right)+s_{T_{j}}\right) .
$$

The function $f_{j}$ is strictly increasing and strictly concave with

$$
\frac{d}{d w_{j, 0}} f\left(w_{j, 0}\right)=\frac{\bar{W}_{j}}{b_{j}} \exp \left(-s_{T_{j}} \Phi^{-1}\left(w_{j, 0} / b_{j}\right)-(1 / 2) s_{T_{j}}^{2}\right) .
$$

The Karush-Kuhn-Tucker conditions for the convex optimization Problem (16) with the additional constraint $w_{j, 0} \leq b_{j}$ for all $j$ are as follows:

$$
\begin{aligned}
& \eta_{1, j} \geq 0, \eta_{2, j} \geq 0, w_{j, 0} \geq 0, \\
& \eta_{1, j} w_{j, 0}=0, \eta_{2, j}\left(w_{0, j}-b_{j}\right)=0, \\
& \sum_{j=1}^{J} w_{j, 0}=0, \\
& -\frac{\delta^{-T_{j}} \bar{W}_{j}}{b_{j}} \exp \left(-s_{T_{j}} \Phi^{-1}\left(w_{j, 0} / b_{j}\right)-(1 / 2) s_{T_{j}}^{2}\right)-\eta_{1, j}+\eta_{2, j}+v=0 .
\end{aligned}
$$

From Eqs. 22 and 25 we obtain:

$$
\eta_{1, t}=v-\frac{\delta^{-T_{j}} \bar{W}_{j}}{b_{j}} \exp \left(-s_{T_{j}} \Phi^{-1}\left(w_{j, 0} / b_{j}\right)-(1 / 2) s_{T_{j}}^{2}\right)+\eta_{2, j} \geq 0
$$


We multiply $\eta_{1, j}$ with $w_{0, j}$ and obtain:

$$
0=w_{j, 0}\left(v-\frac{\delta^{-T_{j}} \bar{W}_{j}}{b_{j}} \exp \left(-s_{T_{j}} \Phi^{-1}\left(w_{j, 0} / b_{j}\right)-(1 / 2) s_{T_{j}}^{2}\right)+\eta_{2, j}\right) .
$$

Using that $\eta_{2, j}\left(w_{j, 0}-b_{j}\right)=0$ we can solve the latter equation for $\eta_{2, j}$ and we obtain:

$$
\eta_{2, j}=-\frac{w_{j, 0}}{b_{j}}\left(v-\frac{\delta^{-T_{j}} \bar{W}_{j}}{b_{j}} \exp \left(-s_{T_{j}} \Phi^{-1}\left(w_{j, 0} / b_{j}\right)-(1 / 2) s_{T_{j}}^{2}\right)\right) \geq 0
$$

which implies

$$
v \leq \frac{\delta^{-T_{j}} \bar{W}_{j}}{b_{j}} \exp \left(-s_{T_{j}} \Phi^{-1}\left(w_{j, 0} / b_{j}\right)-(1 / 2) s_{T_{j}}^{2}\right) .
$$

since $w_{j, 0} \geq 0$. Finally, using $\eta_{2, j}\left(w_{j, 0}-b_{j}\right)=0$ we have

$$
0=-\frac{w_{j, 0}}{b_{j}}\left(w_{0, j}-b_{j}\right)\left(v-\frac{\delta^{-T_{j}} \bar{W}_{j}}{b_{j}} \exp \left(-s_{T_{j}} \Phi^{-1}\left(w_{j, 0} / b_{j}\right)-(1 / 2) s_{T_{j}}^{2}\right)\right) .
$$

If $v>0$, then $w_{j, 0}<b_{j}$. Indeed, if $w_{j, 0}=b_{j}$ and $v>0$ then condition (29) is violated. Moreover, since $w_{j, 0}<b_{j}$, then $\eta_{j, 2}=0$ by the second Slater's condition in (23). Therefore, $w_{0, j}>0$, else condition (26) is violated. It follows from Eq. 30 that $w_{0, j}$ must solve

$$
v-\frac{\delta^{-T_{j}} \bar{W}_{j}}{b_{j}} \exp \left(-s_{T_{j}} \Phi^{-1}\left(w_{j, 0} / b_{j}\right)-(1 / 2) s_{T_{j}}^{2}\right)=0
$$

and after some re-arrangements we obtain

$$
w_{0, j}=b_{j} \Phi\left(-\frac{1}{s_{T_{j}}} \log \left(\frac{v}{\xi_{0} W_{0}}\right)-\frac{1}{s_{T_{j}}}\left(T_{j} \log (\delta)-r T_{j}+\frac{1}{2} s_{T_{j}}^{2}\right)\right) .
$$

If $v<0$, then $\eta_{j, 2}>0$ else condition (26) is violated since the inequality

$$
v-\frac{\delta^{-T_{j}} \bar{W}_{j}}{b_{j}} \exp \left(-s_{T_{j}} \Phi^{-1}\left(w_{j, 0} / b_{j}\right)-(1 / 2) s_{T_{j}}^{2}\right)<0
$$

holds for all $w_{0, j} \in\left[0, b_{j}\right]$. Therefore, $w_{0, j}=b_{j}$ by the second Slater's condition in (23).

If $v=0$ and $w_{0, j} \neq b_{j}$ then $w_{0, j}=0$ by Eq. 30. However, when $w_{0, j}=0$ then Eq. 26 is violated. Thus, also in this case we must have $w_{0, j}=b_{j}$.

\section{References}

1. Barberis, N., Huang, M.: Mental accounting, loss aversion and individual stock returns. J. Financ. 56(4), 1247-1292 (2001)

2. Barberis, N., Huang, M.: The loss aversion/narrow framing approach to the equity premium puzzle. In: Mehra, R. (ed.) Handbook of Investments: The Handbook of the Equity Premium, pp. 199-228. North Holland, Amsterdam (2008)

3. Barberis, N., Huang, M.: Preferences with frames: a new utility specification that allows for framing of risks. J. Econ. Dyn. Control 33(8), 1555-1576 (2009)

4. Barberis, N., Huang, M., Thaler, R.H.: Individual preferences, monetary gambles, and stock market participation: a case for narrow framing. Am. Econ. Rev. 96(4), 1069-1090 (2006)

5. Benartzi, S., Thaler, R.H.: Myopic loss aversion and the equity premium puzzle. Q. J. Econ. 110(1), 75$92(1995)$ 
6. Berkelaar, A.B., Kouwenberg, R., Post, T.: Optimal portfolio choice under loss aversion. Rev. Econ. Stat. 86(4), 973-987 (2004)

7. Bernard, C., Ghossoub, M.: Static portfolio choice under cumulative prospect theory. Math. Financ. Econ. 2(4), 277-306 (2010)

8. Brunel, J.L.: Revisting the asset allocation challenge through a behavioural finance lens. J. Wealth Manag. Fall, 10-20 (2003)

9. Brunel, J.L.: A behavioural finance approach to strategic asset allocation-a case study. J. Invest. Consult. 7(3), 61-69 (2006)

10. Chhabra, A.B.: Beyond markowitz: a comprehensive wealth allocation framework for individuals. J. Wealth Manag. 7(4), 8-34 (2005)

11. Chhabra, A.B., Koneru, R., Zaharoff, L.: Creating a goal-based wealth allocation process. J. Wealth Manag. 11(3), 48-55 (2009)

12. Das, S.R., Markowitz, H., Scheid, J., Statman, M.: Portfolio optimization with mental accounts. J. Financ. Quant. Anal. 45(2), 311-334 (2010)

13. De Giorgi, E.G., Legg, S.: Dynamic portfolio choice and asset pricing with narrow framing and probability weighting. Working paper, Available at http://ssrn.com/abstract=1413087 (2009)

14. Deaton, A., Muellbauer, J.: Economics and Consumer Behavior. Cambridge University Press, Cambridge (1980)

15. Diecidue, E., van de Ven, J.: Aspiration level, probability of success and failure, and expected utility. Int. Econ. Rev. 49(2), 683-700 (2008)

16. Dierkes, M., Erner, C., Zeisberger, S.: Investment horizon and the attractiveness of investment strategies: a behavioral approach. J. Banking Financ. 34(5), 1032-1046 (2010)

17. He, X.D., Zhou, X.Y.: Portfolio choice under cumulative prospect theory: an analytical treatment. Manag. Sci. 57(2), 315-331 (2011)

18. Hens, T., Bachmann, K.: Behavioural Finance for Private Banking, The Wiley Finance Series. Wiley, New York (2008)

19. Jin, H., Zhou, X.Y.: Behavioral portfolio selection in continuous time. Math. Financ. 18(3), 385426 (2008)

20. Kahneman, D., Tversky, A.: Prospect theory: an analysis of decisions under risk. Econometrica 47(2), 263291 (1979)

21. Karatzas, I., Shreve, S.: Methods of Mathematical Finance. Springer, New York (1998)

22. Lopes, L.L.: Between hope and fear: the psychology of risk. Adv. Exp. Soc. Psychol. 20, 255-295 (1987)

23. Nevin, D.: Goal-based investing: integrating traditional and behavioural finance. J. Wealth Manag. 6(4), 823 (2004)

24. Payne, J.W.: It is whether you win or lose: the importance of the overall probabilities of winning or losing in risky choice. J. Risk Uncertain. 30(1), 5-19 (2005)

25. Rieger, M.O.: SP/A and CPT: a reconciliation of two behavioral decision theories. Econ. Lett. 108(3), 327329 (2010)

26. Shefrin, H.: Ending the management illusion: preventing another financial crisis. Ivey Bus. J., JanuaryFebruary (2009)

27. Shefrin, H., Statman, M.: Behavioral portfolio theory. J. Financ. Quant. Anal. 35(2), 127-151 (2000)

28. Statman, M.: Lottery players/stock traders. Financ. Anal. J. 58(1), 14-21 (2002)

29. Tversky, A., Kahneman, D.: Advances in prospect theory: cumulative representation of uncertainty. J. Risk Uncertain. 5(4), 297-323 (1992)

30. Wang, H., Suri, A., Laster, D., Alamdi, H.: Portfolio selection in goal-based wealth management. J. Wealth Manag. 14(1), 55-65 (2011) 